ARTICLE

DOI: $10.1038 / s 41467-018-06948-x$

\title{
Adhesion to nanofibers drives cell membrane remodeling through one-dimensional wetting
}

\author{
Arthur Charles-Orszag (10 1,2, Feng-Ching Tsai ${ }^{3,4}$, Daria Bonazzi ${ }^{1}$, Valeria Manriquez ${ }^{1,2}$, Martin Sachse ${ }^{5}$, \\ Adeline Mallet ${ }^{5}$, Audrey Salles ${ }^{6}$, Keira Melican ${ }^{1,12}$, Ralitza Staneva ${ }^{7}$, Aurélie Bertin ${ }^{3,4}$, Corinne Millien ${ }^{8}$, \\ Sylvie Goussard ${ }^{1}$, Pierre Lafaye ${ }^{9}$, Spencer Shorte ${ }^{6}$, Matthieu Piel (10) 10,11, Jacomine Krijnse-Locker ${ }^{5}$, \\ Françoise Brochard-Wyart ${ }^{3,4}$, Patricia Bassereau (i) ${ }^{3,4} \&$ Guillaume Duménil ${ }^{1}$
}

The shape of cellular membranes is highly regulated by a set of conserved mechanisms that can be manipulated by bacterial pathogens to infect cells. Remodeling of the plasma membrane of endothelial cells by the bacterium Neisseria meningitidis is thought to be essential during the blood phase of meningococcal infection, but the underlying mechanisms are unclear. Here we show that plasma membrane remodeling occurs independently of F-actin, along meningococcal type IV pili fibers, by a physical mechanism that we term 'onedimensional' membrane wetting. We provide a theoretical model that describes the physical basis of one-dimensional wetting and show that this mechanism occurs in model membranes interacting with nanofibers, and in human cells interacting with extracellular matrix meshworks. We propose one-dimensional wetting as a new general principle driving the interaction of cells with their environment at the nanoscale that is diverted by meningococci during infection.

\footnotetext{
${ }^{1}$ Pathogenesis of Vascular Infections Unit, INSERM, Institut Pasteur, Paris 75015, France. ${ }^{2}$ Université Paris Descartes, Sorbonne Paris Cité, Paris 75006 , France. ${ }^{3}$ Laboratoire Physico Chimie Curie, Institut Curie, PSL Research University, CNRS UMR168, Paris 75005, France. ${ }^{4}$ Sorbonne Université, Paris 75005 , France. ${ }^{5}$ Ultrapole, Institut Pasteur, Paris 75015, France. ${ }^{6}$ Imagopole, Institut Pasteur, Paris 75015, France. ${ }^{7}$ Institut Curie, PSL Research University, CNRS, UMR 144, Paris 75005, France. ${ }^{8}$ Paris Cardiovascular Research Center, Paris 75015, France. ${ }^{9}$ Antibody Engineering, Institut Pasteur, Paris 75015 , France. 10 Systems Biology of Cell Polarity and Cell Division, Institut Pierre-Gilles De Gennes, Paris 75005, France. ${ }^{11}$ Institut Curie, Paris 75005, France. ${ }^{12}$ Present address: Department of Neuroscience, Swedish Medical Nanoscience Center, Karolinska Institutet, Solna 171 77, Sweden. Correspondence and requests for materials should be addressed to G.D. (email: guillaume.dumenil@pasteur.fr)
} 
C ontrol of the shape of biological membranes is fundamental for the maintenance of multiple functions in the eukaryotic cell ${ }^{1}$. Acting as the interface of the cell with its surrounding environment, the plasma membrane is a particularly important compartment that is subject to a precise control of its shape and dynamics. Plasma membrane remodeling occurs at very small scales, for example in the biogenesis of caveolae ${ }^{2}$ or during the formation of clathrin coated pits $^{3}$. At larger scales, remodeling of the plasma membrane plays an important role in a wide variety of biological processes, such as the uptake of large particles by phagocytosis ${ }^{4}$ or in the formation of actin-based membrane structures that support cell migration and probing of the extracellular environment, such as filopodia or lamellipodia 5 . In the context of pathological conditions, especially in bacterial, viral and fungal infections, pathogens manipulate the shape of the plasma membrane to enter host cells. This is often achieved by diverting the actin cytoskeleton ${ }^{6-8}$. Other pathogens remain extracellular and must then resist mechanical strains such as those generated by flow ${ }^{9}$. The bacterium Neisseria meningitidis (or meningococcus) is a human pathogen that, while remaining extracellular ${ }^{10}$, massively remodels the host cell plasma membrane to form filopodia-like protrusions that intercalate between aggregated bacteria upon adhesion to the host cell surface. It was shown in vitro that plasma membrane remodeling allows $N$. meningitidis to proliferate on the outside of the host cell while mechanically resisting high shear stress levels ${ }^{11}$, suggesting a central role for plasma membrane remodeling in the blood phase of N. meningitidis pathogenesis where bacteria are subject to high shear. Colonization of the blood vessels by $N$. meningitidis eventually leads to a loss of vascular function that translates into hemorrhagic lesions in organs throughout the body, including the skin where it presents as characteristic purpuric rashes ${ }^{12-14}$. Despite the intensive use of antibiotics, the case fatality rate for meningococcal sepsis can still reach $52 \%{ }^{15}$. Understanding this process is thus important in the study of both infectious processes and mechanisms of plasma membrane dynamics.

The molecular mechanisms by which $N$. meningitidis remodels the host cell plasma membrane are still elusive. While membrane protrusions are enriched in $\mathrm{F}$-actin ${ }^{16}$, our previous work has shown that inhibition of actin polymerization ${ }^{11,16,17}$ or depletion of host cell $\mathrm{ATP}^{17}$ have no effect on the remodeling of the host cell plasma membrane. Bacterial type IV pili (T4P), which are long retractile fibers with a diameter of $6 \mathrm{~nm}$, are required for plasma membrane remodeling in addition to their role in specific adhesion to human cells ${ }^{12,18}$. Indeed, adhesion of non-piliated bacteria mediated by non-fibrillar adhesins, like Opa, does not lead to the formation of plasma membrane protrusions ${ }^{19}$. Furthermore, plasma membrane remodeling is tightly linked to the amount of T4P expressed by the bacteria, as a $30 \%$ decrease in T4P is sufficient to strongly decrease cell surface remodeling ${ }^{20}$. However, the molecular mode of action of $\mathrm{T} 4 \mathrm{P}$ in plasma membrane remodeling is currently unknown.

In this study, we provide evidence that plasma membrane remodeling occurs in vivo inside human blood vessels during colonization by $N$. meningitidis in an animal model of infection. We show that plasma membrane remodeling occurs as discrete and dynamic protrusions at the level of the single bacterium in vitro and that they adhere to individual T4P fibers in a process reminiscent of membrane wetting. We then bring mathematical evidence that membrane wetting can occur on such small fibers, showing that membrane wetting exists in a new regime that we termed one-dimensional wetting, and that adhesion to nanoscale fibers is sufficient to drive membrane remodeling in a reconstituted system. Finally, we show that the ability of nanoscale fibers to drive membrane remodeling is also true for other naturally occurring fibers such as the ones found in native extracellular matrices, suggesting that one-dimensional membrane wetting is a general mechanism for plasma membrane remodeling in human cells.

\section{Results}

$\mathrm{Nm}$ induces plasma membrane remodeling in vivo. Plasma membrane remodeling by meningococcus has only been observed in cultured cells. Therefore, we first sought to verify the existence of plasma membrane remodeling by meningococcus in vivo. Because of the human specificity of meningococcus, we used a recently developed humanized animal model of infection where human dermal vessels in skin are grafted onto immunodeficient mice $^{12}$. Staining of human blood vessels during vascular colonization by $N$. meningitidis revealed that the surface of human endothelial cells was reorganized in the form of plasma membrane protrusions that intercalated between aggregated bacteria (Fig. 1a and Supplementary Figure 1a and b), co-localized with T4P (Fig. 1a), and were enriched in F-actin (Supplementary Figure 1a), paralleling earlier in vitro studies ${ }^{11,16,17}$. This data shows that the endothelial cell plasma membrane is remodeled during vascular colonization by meningococcus in vivo, supporting the idea of a key role of plasma membrane remodeling in meningococcal pathogenesis, and warranting to further study its underlying mechanisms.

Individual bacteria trigger multiple dynamic membrane protrusions. At an early stage of infection, meningococcus adheres to the endothelium as individual bacteria ${ }^{12}$. Scanning electron microscopy (SEM) showed plasma membrane remodeling occurred even at the level of single bacteria as several discrete protrusions surrounding the bacterial body (Fig. 1b). It should be noted that in standard SEM technical conditions used here T4P filaments are not conserved. Live cell oblique fluorescence imaging of endothelial cells expressing a fluorescent plasma membrane marker revealed that these discrete protrusions were elicited immediately upon adhesion of individual bacteria to host endothelial cells and, while remaining in close proximity with the bacterial body, had dynamic properties (Fig. 1c and Supplementary Movie 1). F-actin was not detectable in these protrusions over time ranges of 5-10 $\mathrm{min}$, demonstrated by the absence of LifeAct-mCherry signal (Supplementary Movie 2). Over several bacterial division events, plasma membrane protrusions accumulated in the nascent microcolony as bacteria proliferate on top of the host cell (Supplementary Figure 1c and Supplementary Movie 3). By recording images at high speed, we could track the fate of each plasma membrane protrusion (Fig. 1d-f and Supplementary Movie 5). Individual bacteria induced four to five protrusions on average (Fig. 1e), some with lifetimes as short as 170 milliseconds (Fig. 1f). Within large bacterial aggregates, protrusions were no longer dynamic, and the rare disappearing events were restricted to the edge of the aggregate (Supplementary Figure 1d and Supplementary Movie 4), pointing to a possible maturation process stabilizing the interactions with the host cell. In individual bacteria, the growth and retraction rates of plasma membrane protrusions ( $k_{\mathrm{on}}$ and $k_{\mathrm{off}}$, respectively) were similar, indicating that there was a dynamic equilibrium between appearance and disappearance of the protrusions (Fig. 1g, h). The average lifetime for plasma membrane protrusions, equal to $1 / k_{\text {off }}$, was $44 \pm 7 \mathrm{sec}$ (Fig. $1 \mathrm{i}$ and Supplementary Movie 5). Bacteria induced significantly more plasma membrane protrusions in cells where F-actin was depolymerized with cytochalasin D (CytoD) compared to control cells (Fig. 1j, k and Supplementary Movie 6). This is in line with previous studies demonstrating that plasma membrane remodeling by meningococcus does not depend on actin polymerization ${ }^{11,16,17}$. Plasma membrane protrusions in 
CytoD-treated cells also showed significantly shorter lifetimes (Fig. 11), further suggesting that F-actin stabilizes plasma membrane protrusions once they are formed and pointing to a role of membrane tension in this remodeling. Isogenic bacteria deficient in pilus retraction were still capable of inducing plasma membrane protrusions (pilT mutant, Fig. $1 \mathrm{~m}, \mathrm{n}$ and Supplementary Movie 7), showing that forces exerted by pilus retraction were not required to remodel the host cell plasma membrane, as previously proposed for large bacterial aggregates ${ }^{17}$. Pilus retraction- deficient bacteria elicited discrete protrusions (Fig. 1n and Supplementary Figure 2), as well as ring-like structures (Fig. 1m, o), reflecting more protrusions that are not resolved optically and larger protrusions (Supplementary Figure 2) likely due to the higer amount of T4P produced by the pilT mutant. Strikingly, discrete membrane protrusions induced by pilT were no longer dynamic. Once they were formed, they never disappeared during the time of observation (Fig. 1p), suggesting a causal link between pilus retraction and disappearance of plasma membrane a
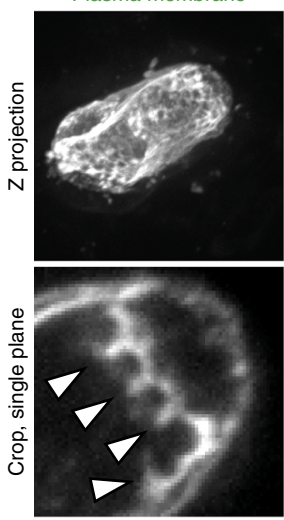

C Time (s):
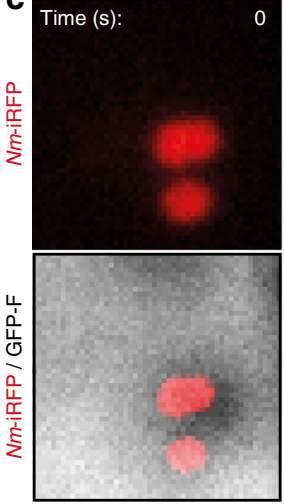

d
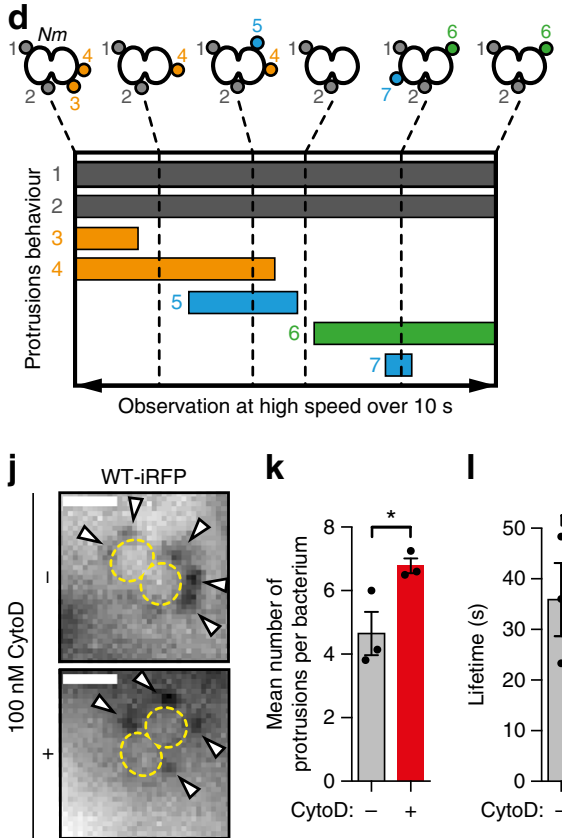
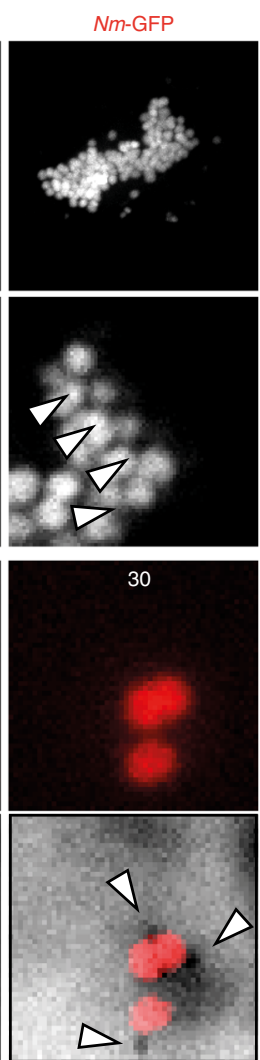

$\triangle$

$\mathbf{k}$

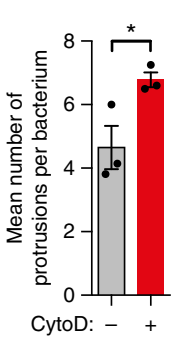

I

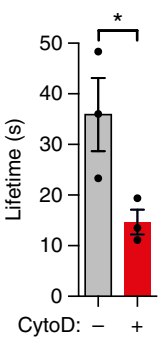

Type IV pili
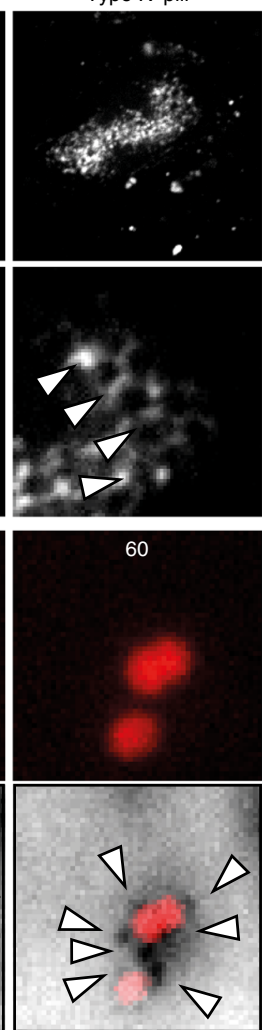

e

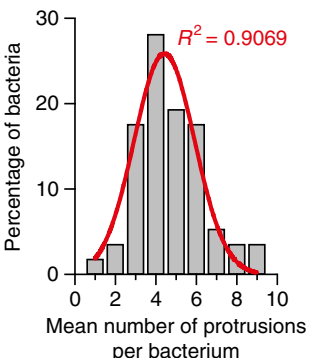

m

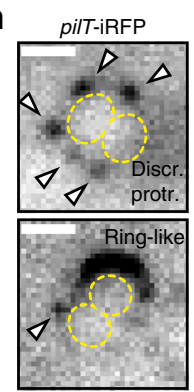

Merge+DAPI
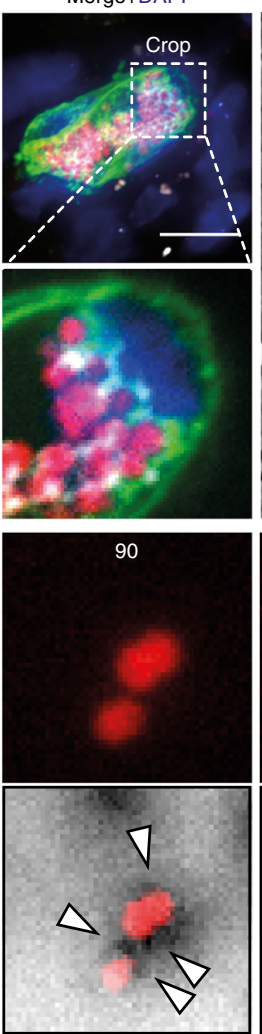

f

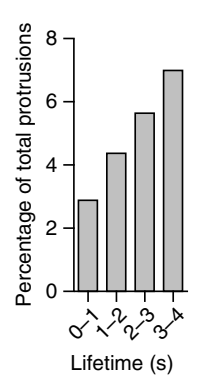

n

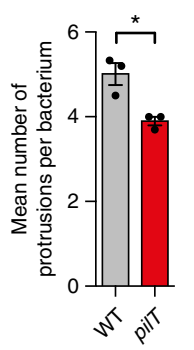

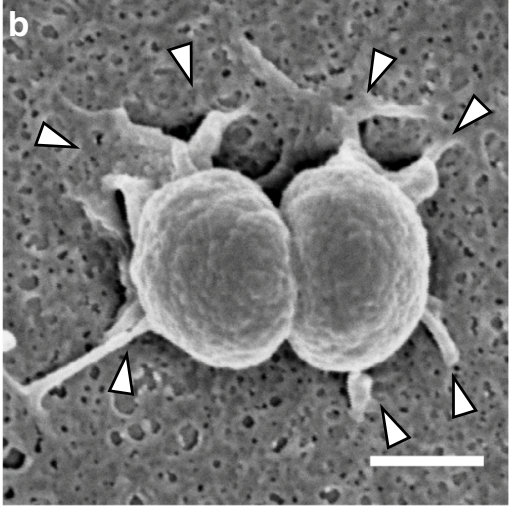

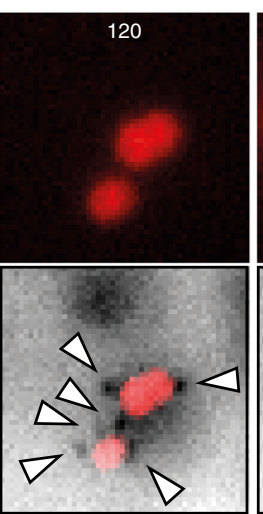

g

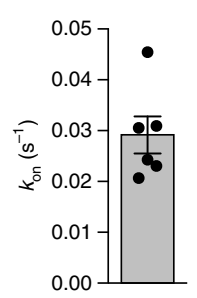

h
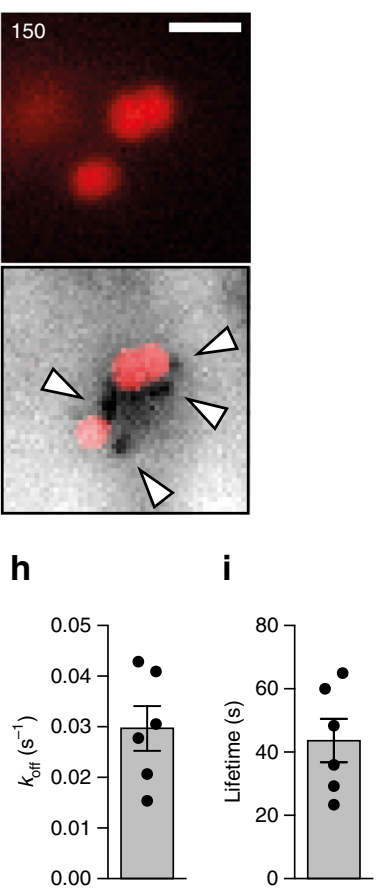

o
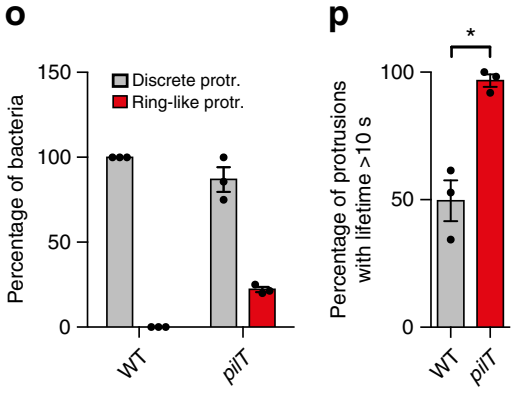
Fig. 1 Plasma membrane remodeling by $\mathrm{Nm}$ occurs in vivo, and is initiated at the level of the individual bacterium in vitro. a Histoimmunolabeling of human blood vessels in a mouse after $3 \mathrm{~h}$ of infection with $\mathrm{N}$. meningitidis $(\mathrm{Nm})$-GFP showing plasma membrane protrusions co-localizing with T4P between aggregated bacteria (arrowheads). Scale bar, $10 \mu \mathrm{m}$. Representative of $n=2$ mice. b Scanning electron micrograph of an endothelial cell infected for $10 \mathrm{~min}$. Arrowheads show plasma membrane protrusions. Scale bar, $500 \mathrm{~nm}$. Representative of $n=2$ independent experiments performed in duplicate. c Oblique illumination live imaging of an endothelial cell expressing the membrane marker GFP-F (GFP-Farnesyl, inverted contrast) infected by individual $\mathrm{Nm}$-iRFP. Arrowheads show dynamic plasma membrane protrusions. Scale bar, $2 \mu \mathrm{m} . n>10$ independent experiments. d Types of plasma membrane protrusions observed in high speed oblique illumination imaging. e Frequency distribution (gray bars) and Gaussian fit (red line) of the number of protrusions per bacterium. $\mathbf{f}$ Frequency distribution of the number of plasma membrane protrusions with short observed lifetimes. $\mathbf{g}$-i Plasma membrane protrusions on-rate $\left(k_{\text {on }}\right)$, off-rate $\left(k_{\text {off }}\right)$ and mean lifetime. Gray bars and error bars represent the mean \pm SEM over 57 bacteria in six independent experiments. Dots represent the mean values of 4-14 bacteria in each experiment. j-I Plasma membrane protrusions are still induced by $\mathrm{Nm}$ in cytochalasin D-treated cells but have shorter lifetimes. Scale bars, $2 \mu \mathrm{m}$. Bars and error bars in graphs represent the mean \pm SEM in three independent experiments. Dots represent the mean of each individual experiment. The total number of bacteria analyzed was 26 and 15 in non-treated and treated cells, respectively. $\mathbf{m}-\mathbf{p}$ Mutant bacteria deficient for pilus retraction trigger plasma membrane protrusions with altered morphologies and that are no longer dynamic. Scale bars, $2 \mu \mathrm{m}$. Bars and error bars in graphs represent the mean \pm SEM of three independent experiments. Dots represent the mean of each individual experiment. The total number of bacteria analyzed was 31 WT-iRFP and 28 pilT-iRFP

protrusions. When taken together, these observations prompted us to hypothesize that plasma membrane protrusions are induced by a direct interaction of the plasma membrane with T4P fibers.

T4P fibers dictate the geometry of plasma membrane remodeling. As a first evidence, 3D Structured Illumination Microscopy showed that plasma membrane protrusions were intertwined with T4P fibers within pairs of meningococci (Supplementary Figure $3 \mathrm{a}$ and $\mathrm{b}$ ) and larger bacterial aggregates (Supplementary Figure $3 c$ and d). Next, to gain ultrastructural information, we performed scanning electron microscopy after stabilization of T4P, which are otherwise fragile, by coating them with a monoclonal antibody. In these conditions, numerous fibershaped structures were visible (Fig. 2a, compare with Fig. 1b). These fibers stained positive for T4P in immunogold labeling assays (Fig. 2b) and showed direct association with plasma membrane protrusions (Fig. 2a, b). While the majority of T4P fibers formed a dense meshwork around bacterial bodies (Supplementary Figure 4a), some other extended further away from bacteria and were as long as $20 \mu \mathrm{m}$ (Supplementary Figure $4 \mathrm{~b}$ ). However, plasma membrane protrusions were only seen associated with T4P in the vicinity of bacterial bodies, and not with T4P fibers adhering flat to the plasma membrane (Supplementary Figure $4 \mathrm{c}$ ). At longer times, $2 \mathrm{~h}$ post-infection, high pressure freezing and freeze substitution prior to transmission electron microscopy revealed that plasma membrane protrusions were embedded in a dense meshwork-like structure that occupied the space between adjacent bacteria in the microcolony (Fig. 2c and Supplementary Figure 5a). This meshwork was sometimes present around bacteria with no neighboring bacterium and still contained plasma membrane protrusions (Supplementary Figure 5b). Immunogold labeling of T4P confirmed that this meshwork was made of T4P fibers (Fig. 2d). It should be emphasized that $2 \mathrm{~h}$ after the initial contacts between pili and host cells bacteria have proliferated, the cells have moved and reorganized their cytoskeleton thus generating a complex picture that has been visualized in Neisseria gonorrhoeae ${ }^{21}$.

Nevertheless, these data point to a scaffolding mechanism for plasma membrane remodeling exerted by T4P fibers. Several lines of evidence support this hypothesis. First, scanning electron microscopy of bacterial cultures showed that the T4P meshwork pre-existed adhesion with host cells (Fig. 2e). Second, adhesion of non-piliated bacteria to endothelial cells mediated by a nonfibrillar outer membrane adhesin, Opa, and its cellular receptor, CEACAM1, led to a phagocytic cup-shaped remodeling of the plasma membrane instead of discrete protrusions (Fig. 2f). SEM images show bacteria nearly engulfed in plasma membrane.
Furthermore, re-expression of $\mathrm{T} 4 \mathrm{P}$ reverts plasma membrane reorganization to dot-like protrusions, demonstrating a geometrical effect of T4P fibers on the shape of plasma membrane remodeling (Fig. 2f). Both membrane remodeling processes were again independent of F-actin polymerization (Supplementary Figure 6). Finally, presence of an anti-T4P antibody during the infection of endothelial cells strongly impaired the ability of bacterial microcolonies to remodel the plasma membrane in a dose-dependent manner (Fig. 2g, h), suggesting that plasma membrane remodeling by meningococcus depends on the adhesive properties of T4P fibers. Therefore, we propose that the remodeling of the plasma membrane is driven by adhesion forces between receptors in the membrane bilayer and T4P fibers. The pilin monomer which is the main constituent of TFP was shown to interact with a heterodimer formed by CD147 and the $\beta 2$-adrenergic receptor ${ }^{22}$.

Physical model of membrane remodeling driven by wetting on nanofibers. We hypothesized that membrane remodeling occurs by membrane wetting along T4P fibers and explored the physical processes involved. It is known that a liposome spreads onto micrometer-scale adhesive glass fibers ${ }^{23}$. In this case, the free energy $F_{2 \mathrm{D}}$ of the phospholipid bilayer spreading on a fiber of radius $r$ is the sum of three contributions: (i) the curvature energy of the bilayer, (ii) the surface energy of the bilayer and (iii) the gain of adhesion energy between the bilayer and the fiber: $F_{2 \mathrm{D}}=\frac{\pi \kappa_{\mathrm{b}} L}{r}+2 \pi r \sigma L+2 \pi r(-W) L$, where $\kappa_{\mathrm{b}}$ and $\sigma$ are the bending modulus and surface tension of the bilayer, respectively, $L$ is the spreading length of the membrane along the fiber, and $W$ is the adhesion energy of binding of mobile receptors on the membrane to ligands on the fiber. From $F_{2 \mathrm{D}}$, we derived the driving force of the spreading of the bilayer, $f_{\mathrm{d}}=2 \pi r\left(W-\frac{\kappa_{\mathrm{b}}}{2 r^{2}}-\sigma\right)$. For the bilayer to spread, $f_{\mathrm{d}}$ has to be positive. Therefore, $f_{\mathrm{d}}=0$ defines a minimal value of the radius $r_{\min }=\sqrt{\frac{\kappa_{\mathrm{b}}}{2(W-\sigma)}}$. In conclusion, the bilayer spreads and wraps around the fiber, a wetting process that is already well known, but only when $f_{\mathrm{d}}>0$, i.e. when $r>r_{\text {min }}$ (Fig. 3a).

The case of $r<r_{\min }$, which would correspond to spreading on a nanofiber, was not reported before. In this case, the bilayer cannot wrap around the fiber because the curvature energy becomes too high and a different scenario is predicted by the model: a membrane nanotube grows on top of the fiber, provided that the membrane tension is low enough (Fig. 3b). In this case, the free energy $F_{1 \mathrm{D}}$ of a membrane tube of radius $R_{\mathrm{t}}$ and length $L_{\mathrm{t}}$ growing along an adhesive fiber is the sum of the tube energy and the gain of the adhesion energy $W_{t} L_{t}$, 
Host cell plasma membrane /
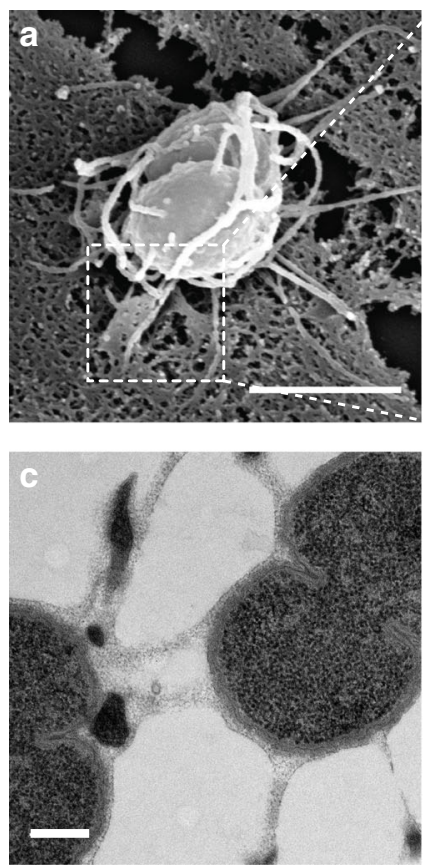

e

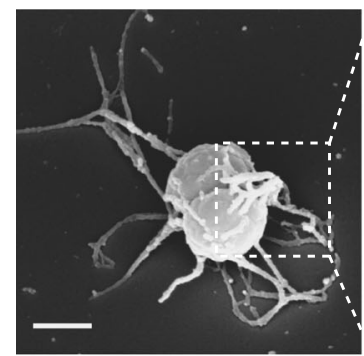

f

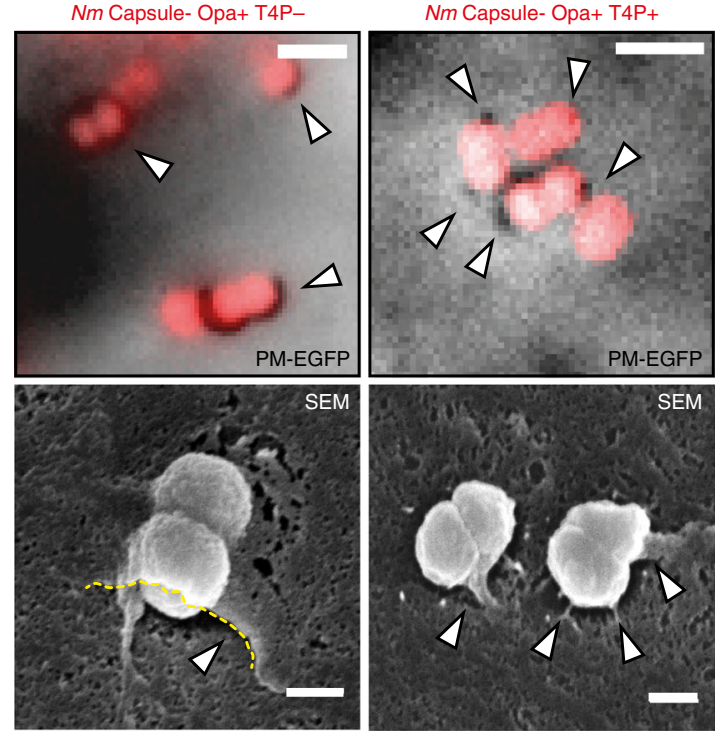

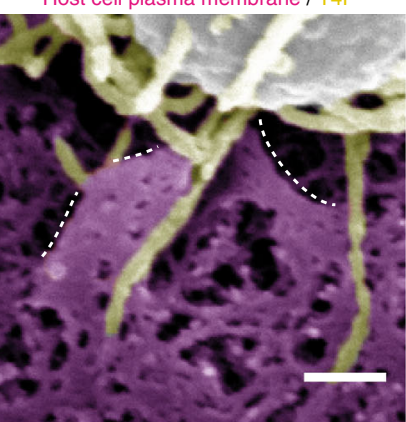

$\mathrm{Nm} /$ Host cell plasma membrane /

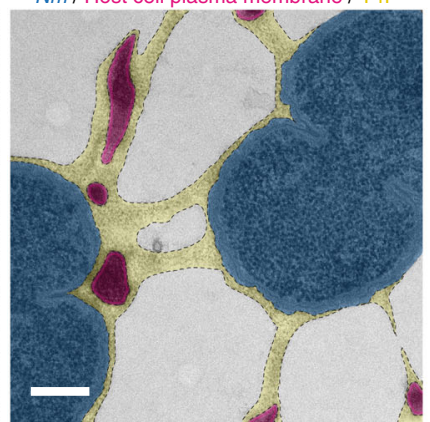

A

g

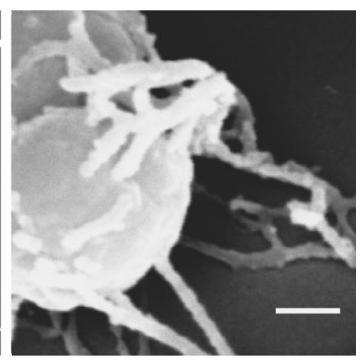

Nm Capsule- Opa+ T4P+

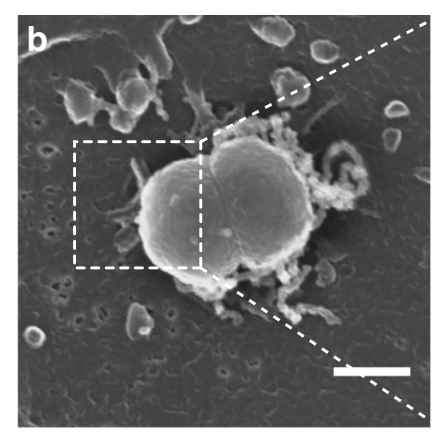

$\circ \mathrm{T} 4 \mathrm{P}(15 \mathrm{~nm}$ gold $)$
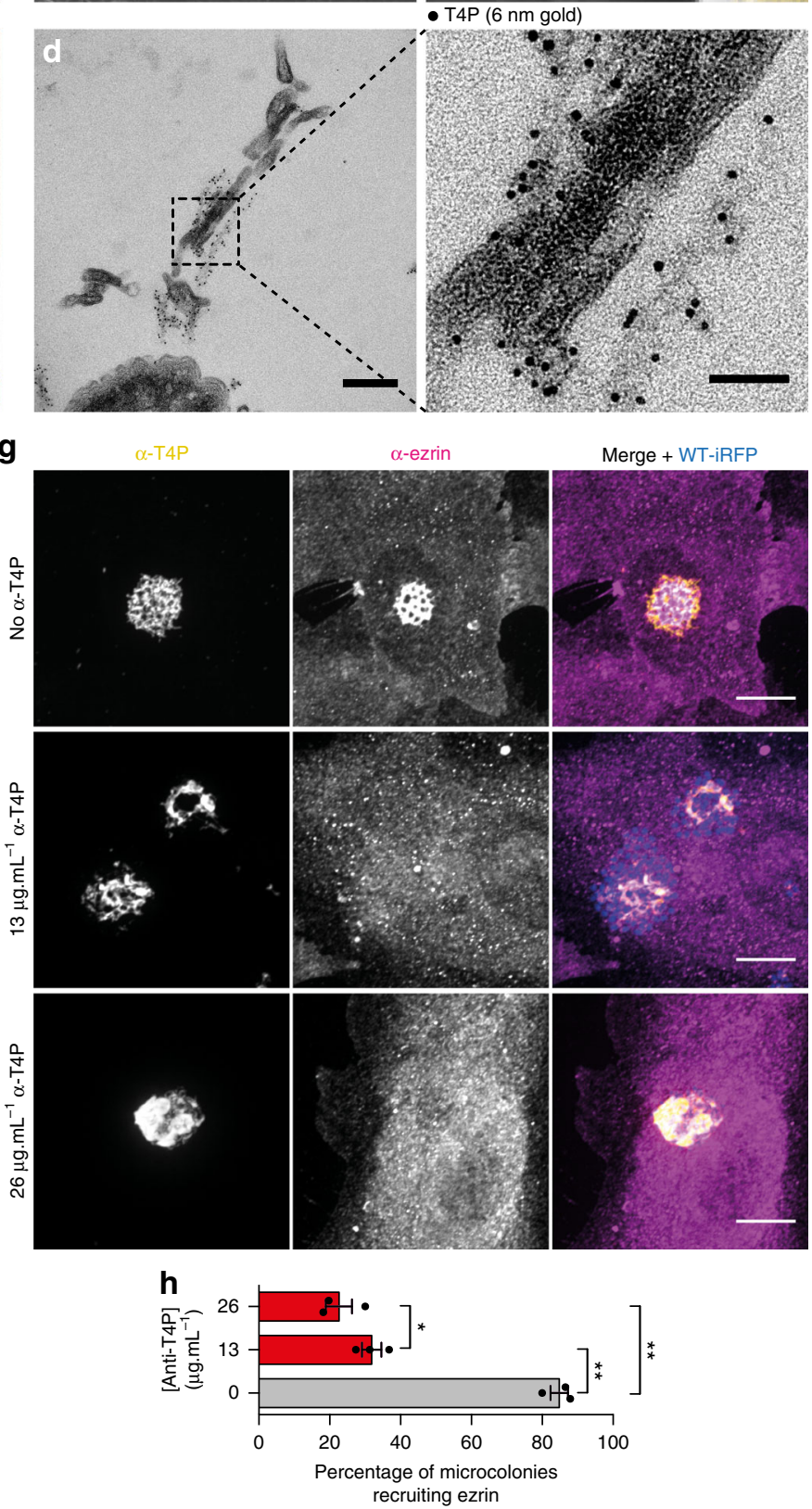

term is the retraction force acting on the tube while the second term is the driving force due to the adhesion along the fiber. If $f_{L}>$ 0 , i.e. when the surface tension $\sigma$ of the membrane is sufficiently low, a membrane nanotube can grow along the fiber. To summarize, our model predicts that, for a given adhesion energy between receptors in the phospholipid bilayer and an adhesive nanotube, $f_{L}=-2 \pi \sqrt{2 \kappa_{\mathrm{b}} \sigma}+W_{\mathrm{t}}$ (See Methods). Here, the first where $U$ is the ligand-receptor binding energy and $\Gamma_{1}$ is the
number of ligands on the fiber per unit length. From the free
energy $F_{1 \mathrm{D}}$, we derived the driving force acting on the membrane

$F_{1 \mathrm{D}}=\frac{\pi \kappa_{\mathrm{b}} L_{\mathrm{t}}}{R_{\mathrm{t}}}+2 \pi R_{\mathrm{t}} \sigma L_{\mathrm{t}}-W_{\mathrm{t}} L_{\mathrm{t}}$. Here, the two first terms are the membrane curvature energy and the surface energy; $W_{\mathrm{t}}=\Gamma_{\mathrm{l}} \cdot U$, where $U$ is the ligand-receptor binding energy and $\Gamma_{1}$ is the 
Fig. 2 The morphology of plasma membrane protrusions is dictated by the fiber-like morphology of T4P. a Scanning electron micrograph of an endothelial cell after 10 min infection and stabilization of the T4P with a monoclonal antibody. Colorized crop shows plasma membrane protrusions (magenta, dotted lined) attached alongside T4P fibers (yellow). Scale bars, $1 \mu \mathrm{m}$ and $200 \mathrm{~nm} . n=2$ independent experiments performed in duplicate. b Same experiment after immunogold labeling of T4P. Protr., protrusions. Scale bars, $500 \mathrm{~nm}$ and $200 \mathrm{~nm} . n=2$ independent experiments performed in duplicate.

c Transmission electron micrograph of a microcolony of $\mathrm{Nm}$ after high pressure freezing and freeze substitution, showing plasma membrane protrusions embedded in a meshwork of T4P. Scale bars, $200 \mathrm{~nm}$. Representative of multiple microcolonies in $n=1$ experiment. d Same experiment after immunogold labeling of T4P. Scale bars, $200 \mathrm{~nm}$ and $50 \mathrm{~nm}$. Representative of multiple microcolonies in $n=1$ experiment. e Scanning electron micrograph of the meshwork of T4P produced by an individual bacterium in culture. Scale bars, $500 \mathrm{~nm}$ and $200 \mathrm{~nm} . n=2$ experiment. f Non-piliated Opa + bacteria remodel the plasma membrane in a phagocytic cup-like fashion. Re-expression of T4P reverts the remodeling to a protrusion morphology. Scale bars, $2 \mu \mathrm{m}$ (darkfield) and $500 \mathrm{~nm}$ (SEM). $n=3$ and 1 experiments. $\mathbf{g}$ An anti-T4P moclonal antibody affects plasma membrane remodeling by Nm. Plasma membrane recruitment was assessed by the accumulation of ezrin. Scale bars, $10 \mu \mathrm{m}$. Representative of $n=3$ experiments. $\mathbf{h}$ Quantification of the experiment in g. Bars and error bars represent the mean \pm SEM. Dots represent the mean of individual experiments. 216 to 240 microcolonies were counted per condition
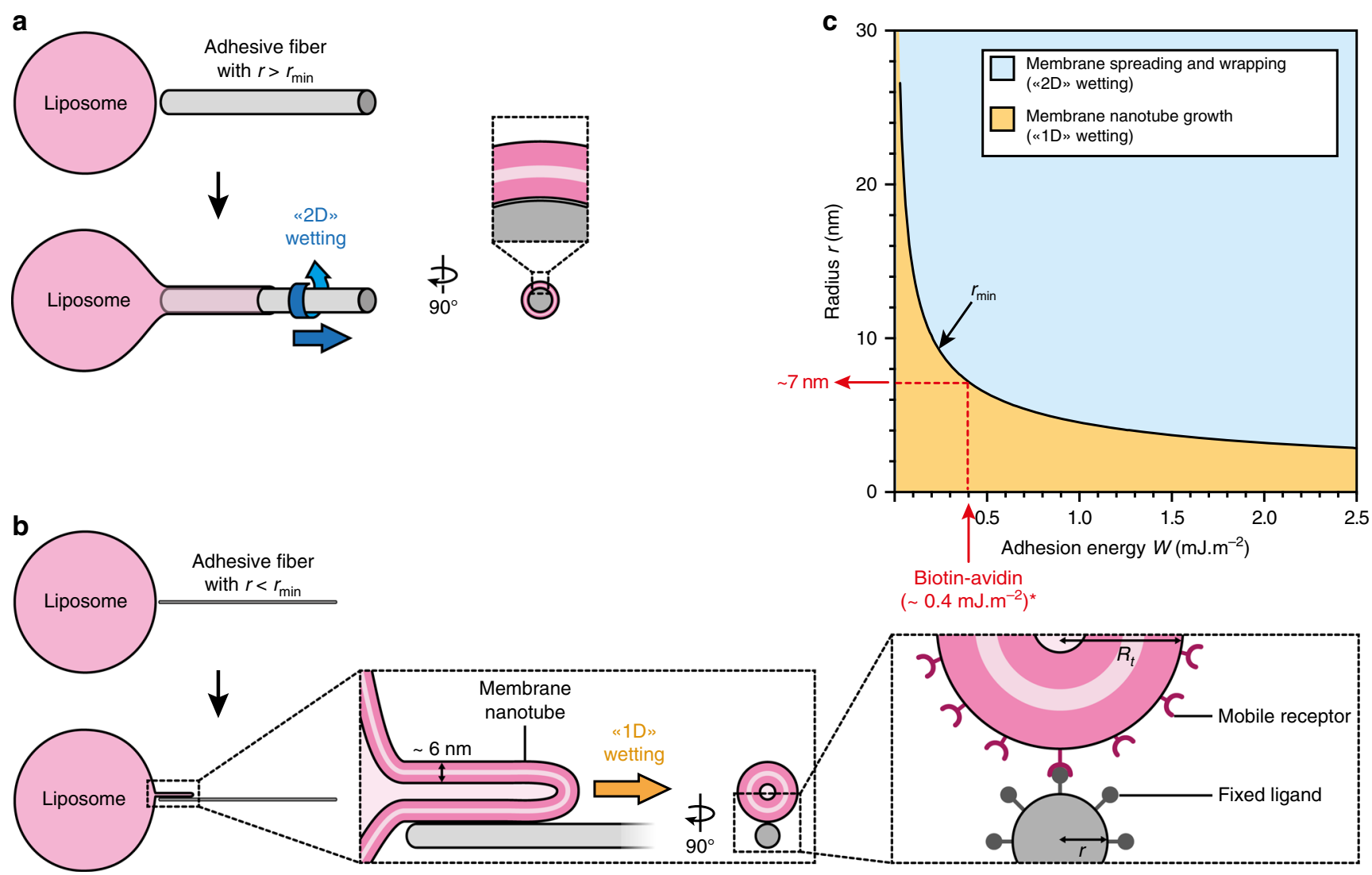

Biotin-avidin $\left(\sim 0.4 \mathrm{~mJ} \cdot \mathrm{m}^{-2}\right)^{\star}$

Fig. 3 Theoretical prediction of a novel regime of wetting for the spreading of a liposome on an adhesive nanofiber. a A liposome spreads on a fiber of radius $r>r_{\min }$ by "2D" membrane wetting, where the bilayer wraps around the fiber. $\mathbf{b}$ On a fiber of radius $r<r_{\text {min, }}$ the liposome cannot wrap the fiber because of the curvature energy of the bilayer and is predicted to grow a tube of radius $R_{\mathrm{t}}$ along the fiber, which we call "1D" membrane wetting. In the inset most to the right mobile receptors and fixed ligands are indicated schematically, their size or distribution are not drawn to scale. $\mathbf{c}$ Phase diagram of a membrane bilayer spreading on a fiber versus nanofiber radius $r$ and adhesion energy $W$. The black line corresponds to $r_{\text {min }}$ separates the "2D" (blue region) and "1D" (yellow region) membrane wetting regimes. $\left(^{*}\right)$ Considering a biotinylated actin filament decorated with NeutrAvidin and a molar ratio of biotinylated actin:actin of 1:10 (Fig. 4), we estimated the adhesion energy $W$ for the biotin-avidin complex to be 0.4 mJ.m-2, yielding a minimum radius $r_{\min }=7 \mathrm{~nm}$ below which "2D" wetting can no longer occur (See Methods)

fiber, there is a minimum radius $r_{\min }$ of the fiber above which the bilayer should spread by canonical membrane wetting ("2D") on the fiber, and below which the bilayer should spread by forming a nanotube along the fiber ("1D"). This last regime of tube formation is very different from other mechanisms of tube formation where tubes are actively pulled by molecular motors $^{24,25}$ or by external mechanical forces ${ }^{26-29}$. For 1D wetting, the driving force is provided by the gain of adhesion energy. We show in Fig. $3 \mathrm{c}$ the phase diagram of membrane nanofiber wetting in the coordinates $r$ (fiber radius) and $W$ (adhesion energy). The two regimes "2D" versus " $1 \mathrm{D}$ " are separated by the curve $r_{\min } \cong \sqrt{\frac{\kappa_{\mathrm{b}}}{2 W}}$ in the limit $\sigma$ «W. The dynamics of protrusions for $1 \mathrm{D}$ and $2 \mathrm{D}$ wetting is ruled by the balance between the driving force discussed above and the drag force associated to the flow of the lipids (See Methods). T4P fibers with their extremely small radius of $3 \mathrm{~nm}$ can thus potentially function in the " $1 \mathrm{D}$ " membrane wetting regime. Indeed, in the 
context of live cells where membrane rigidity is higher $\left(\kappa_{\mathrm{b}} \approx 50 k_{\mathrm{B}} T^{30}\right)$, the adhesion energy necessary for “ $2 \mathrm{D}$ ” wetting, defined by $r_{\text {min }}=r_{\text {pilus }}=3 \mathrm{~nm}$, is extremely large, of order $10 \mathrm{~mJ}$. $\mathrm{m}^{-2}$, which means that for T4P fibers we expect " $1 \mathrm{D}$ " wetting to occur (Supplementary Figure 7). This is supported by scanning electron micrographs showing plasma membrane protrusion along T4P fibers (Fig. 2a, b).

Nanofibers drive membrane remodeling in a model system and in cells. To test this theoretical model in vitro the use of purified pili and lipid vesicles was complicated by the necessity to insert a multimeric protein receptor in the lipid vesicles ${ }^{22}$. We rather used an assay consisting of model membranes (biotinylated giant unilamellar vesicles (GUVs) of typically $20 \mu \mathrm{m}$ diameter) interacting with model nanofibers consisting of biotinylated actin fibers decorated with NeutrAvidin and immobilized on a solid substrate (Fig. 4a). The diameter of single actin filaments, $7 \mathrm{~nm}$, is similar to the diameter of T4P fibers. Confocal microscopy showed that biotinylated GUVs interacting with NeutrAvidincoated filaments deformed along them in two different ways. On thick adhesive bundles, GUVs spread and wrapped around the fibers (Fig. 4b), similar to previous studies of GUVs spreading on glass microfibers ${ }^{23}$ and consistent with the canonical " $2 \mathrm{D}$ " membrane wetting described in our phase diagram (Fig. 3c). However, on thin adhesive nanofibers, GUVs tended to extend thin protrusions aligned along these fibers (Fig. 4c), consistent with the regime of membrane nanotube elongation that is predicted to occur on thin nanofibers (Fig. 3c). Cryo-electron microscopy experiments demonstrated that when phospholipid vesicles were mixed with adhesive nanofibers, membrane nanotubes were clearly in close apposition with the fibers rather than wrapped around them (Supplementary Figure 8). Strikingly, membrane protrusions branched when encountering branched adhesive fibers (Fig. 4c), showing that the shape of the bilayer was governed by the architecture of the adhesive fibers. Interestingly, by taking reported values of the adhesion energy between biotin and avidin $^{31,32}$, we found that the minimum radius $r_{\text {min }}$ below which the membrane should spread on these adhesive nanofibers by nanotube elongation is approximately $7 \mathrm{~nm}$ (Fig. 3c, and see Methods), in agreement with our experimental observations.

Interaction with the extracellular matrix is another situation in which cells interact with nanofibers. To further validate our predictions in the context of the interaction of human cells with components of the extracellular matrix, we investigated how the plasma membrane of endothelial cells deforms on an RGD peptide-coated nanostructured solid substrate. To do so, endothelial cells were cultured on anodized aluminum oxide foils featuring $100 \mathrm{~nm}$ pores with pore walls of less than $15 \mathrm{~nm}$ mimicking fibers of the extracellular matrix (Fig. 4d, e). To reconstitute integrin-fibronectin adhesion without altering the nano-topography of the substrate, we sequentially coated the substrate with an azide functionalized PLL-PEG (azido-(poly-Llysine-graft-poly[ethylene glycol], or APP) and a reactive RGD peptide (BCN-RGD) ${ }^{33}$. Scanning electron microscopy showed that the plasma membrane at the cell contour deformed periodically along the pore walls (Fig. 4d). Filopodia were also seen to follow the complex geometry of the pore walls along their whole length (Fig. 4e). Interestingly, the plasma membrane along filopodia also seemed to deform periodically when encountering a pore wall. To test if protrusions formed on natural ECM nanofibers we performed scanning electron microscopy on native basal membranes extracted from mouse mesentery (Fig. 4f). The extracellular matrices exhibited bundles of fibers of a few tens or hundreds of nanometers, but also fibers down to a few nanometers. When cultured on such matrices, human endothelial cells showed nanoscale plasma membrane protrusions that aligned onto fibers of 5 to about $30 \mathrm{~nm}$ (Fig. $4 \mathrm{~g}$ ). Thus, this set of experiments shows that cell membranes can form protrusions when adhering on naturally occurring nanofibers such as those found in the ECM.

\section{Discussion}

Taken together, these data show that adhesive nanofibers can drive the remodeling of biological membranes at the nanoscale because of a wetting phenomenon that falls into a new regime that we named " $1 \mathrm{D}$ " membrane wetting. In the context of plasma membrane remodeling induced by $N$. meningitidis in human endothelial cells, we propose that adhesion to T4P fibers drives plasma membrane deformation by this mechanism (Fig. 5). This is consistent both with the fact that the process is F-actin independent ${ }^{11,16,17}$, and with the observation that actin polymerization occurs subsequent to membrane deformation ${ }^{17}$. A similar sequence is observed in membrane nanotube pulling experiments in which actin polymerizes in membrane nanotubes initially devoid of F-actin ${ }^{34,35}$.

Calculations based on our model indicate that the adhesion energy between T4P and the cell membrane should be smaller than $10 \mathrm{~mJ} \cdot \mathrm{m}^{-2}$ and the membrane tension should be smaller than a threshold value $\left(\sigma_{t}\right)$ corresponding to $f_{L}=0$ to be in the " $1 \mathrm{D}$ " regime. Assuming pilin monomers are arranged in a helical pitch of $7 \mathrm{~nm}$ in the pilus fiber ${ }^{36}$, an estimation of the binding energy per pair of pilin monomer-cell receptor leads to a minimal value of approximately $100 k_{\mathrm{B}} T$ for " $2 \mathrm{D}$ ” wetting to occur. As a comparison, it can be estimated from atomic force microscopy measurements on Pseudomonas aeruginosa T4P that the binding energy is smaller, approximately $18 k_{\mathrm{B}} T$ (see Methods), which leads to an estimate of the driving force $W_{\mathrm{t}}$ in the order of $10 \mathrm{pN}$. Thus, membrane wetting on T4P is likely to enter the " $1 \mathrm{D}$ " regime, provided that the tube retracting force is smaller than $W_{\mathrm{t}}$. The retraction forces of tubes have been measured for different cell types adhering on substrates with various techniques and are in the range of a few to $20 \mathrm{pN}^{37-39}$. Available estimates of key physical parameters are thus in agreement with the occurrence of "1D" wetting during N. meningitidis interaction with host cells.

Membrane deformation as discrete protrusions along T4P fibers could be seen as a way to prevent the formation of a continuous membrane cup, as seen for the zipper mechanism employed by Listeria monocytogenes or Yersinia spp. were receptor containing plasma membrane adheres to outer membrane invasins ${ }^{40,41}$, leading to bacterial engulfment. Therefore, "1D" wetting along filamentous adhesins could be a strategy to prevent efficient internalization into host cells. Of note, many prokaryotic species, including numerous human pathogens, also produce filamentous appendages which organize into dense meshworks of fibers ${ }^{42-53}$ and which could possibly use a "1D" membrane wetting mechanism in the interaction with their environment or their immobilization on host cells.

Membrane nanotubes were already described to play important roles in other physiological as well as pathological cellular processes $^{54-58}$. Our study therefore describes a new mechanism of membrane nanotubes formation that is central to the interaction between an infectious bacterium and its host cell, but also to normal cell physiology. Indeed, in eukaryotic multicellular organisms, cells interact with proteins of the extracellular matrix that exist as fibers with adhesive properties, such as collagen, fibrillin or fibronectin, with reported diameters as small as 10 $\mathrm{nm}^{59}$. Our data show that fibers of the extracellular matrix can be even smaller, down to $5 \mathrm{~nm}$. Importantly, cells form plasma membrane protrusions on such nanofiber networks. Thus, it will be of interest to assess whether such an F-actin-independent "1D" 

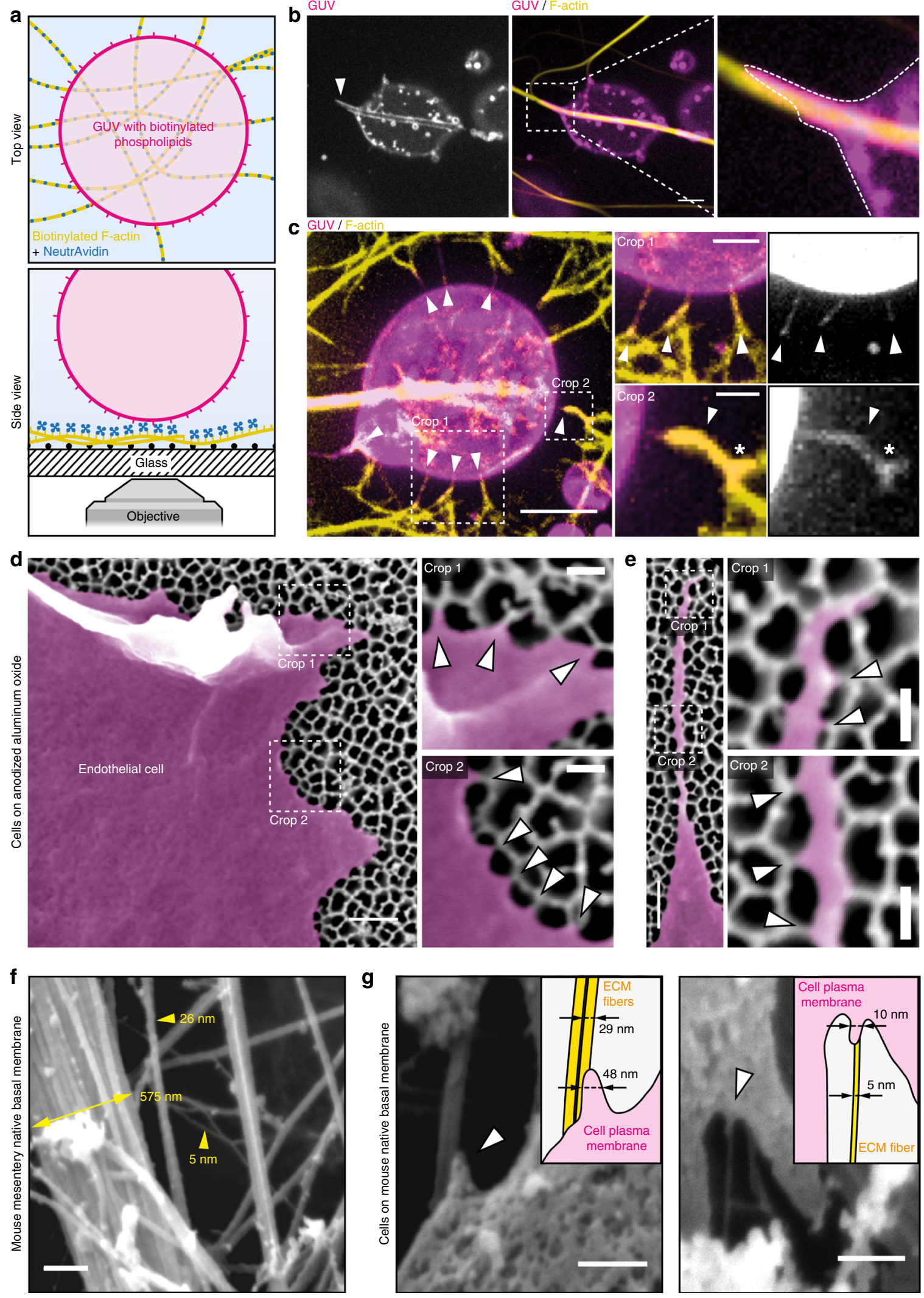

membrane wetting phenomenon plays a role in cell migration through complex environments occurring during diverse biological processes such as development, immune response or cancer.

Our study highlights the notion that cells react to the topography of their environment at the nanoscale ${ }^{60,61}$. It is likely that the development of high resolution light and electron imaging techniques will help uncover the cell response to nano-topographical cues, in particular in the context of the interaction with complex fibrous environments. 
Fig. 4 Membrane wetting on fibers drives the deformation of giant unilamellar vesicles and occurs in human endothelial cells. a Schematic of the experimental setup where giant unilamellar vesicles (GUVs) containing biotinylated phospholipids adhere to biotinylated F-actin fibers decorated with NeutrAvidin. b GUVs spread on thick adhesive bundles (arrowhead) by wrapping around the fibers (crop, dotted line), as predicted by the canonical "2D" membrane wetting regime. Scale bar, $5 \mu \mathrm{m}$. c On thin adhesive fibers, GUVs extend small membrane tubes that align with the fibers (arrowheads), as predicted in the "1D" membrane wetting regime. Membrane tubes can branch when encountering branched adhesive fibers (crop 2, asterisk). Scale bars, 5, 2 and $1 \mu \mathrm{m}$. Representative of $n=3$ experiments. $\mathbf{d}$ Endothelial cells cultured on porous aluminum filters coated with an RGD peptide show local linear plasma membrane deformations that match with the thin pore walls (arrowheads). e Pore walls dictate the path followed by filopodia, where the plasma membrane is also found to deform locally when encountering a pore wall (arrowheads). Representative of $n=2$ experiments. Scale bars, $500 \mathrm{~nm}$ and $150 \mathrm{~nm}$ for the crops. $\mathbf{f}$ SEM shows that native basal membranes from mouse mesentery feature fibers with diameters down to $5 \mathrm{~nm}$. Scale bar, $250 \mathrm{~nm}$. $\mathbf{g}$ Endothelial cells cultured on such extracellular matrices show nanoscale plasma membrane protrusions aligning on nanoscale fibers. Scale bars, 250 and $200 \mathrm{~nm}$

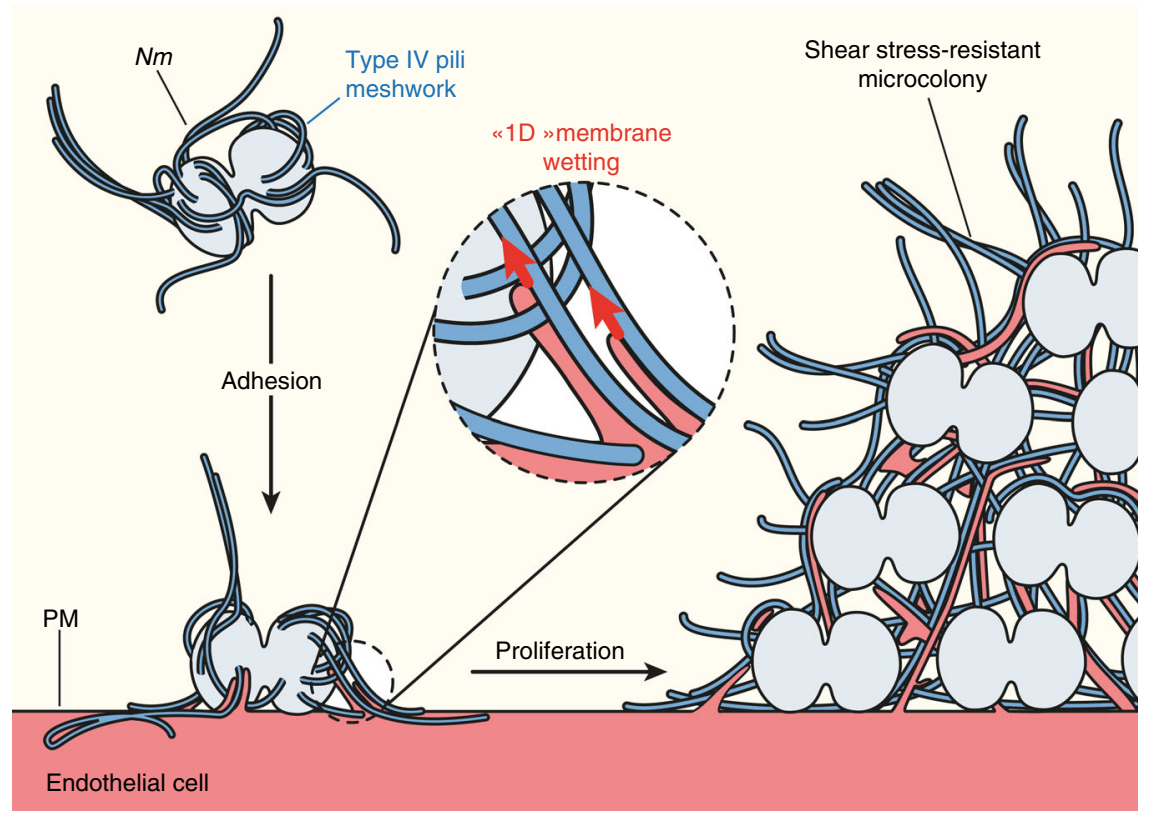

Fig. 5 Working model for the mechanism of wetting-induced plasma membrane protrusions by Nm T4P fibers. Nm produces T4P as a meshwork of fibers. Upon adhesion to the host endothelial cell, the high adhesiveness of T4P allows "1D" membrane wetting of the plasma membrane, and thus remodeling of the membrane alongside T4P fibers. As the bacteria proliferate and aggregate extracellularly, plasma membrane protrusions remain attached to T4P fibers and end up embedded in a dense extracellular T4P meshwork. This complex T4P-plasma membrane structure provides the microcolony with enough mechanical coherence to resist blood flow-generated shear stress

\section{Methods}

Ethics statement. All experimental procedures involving animals were conducted in accordance with guidelines established by the French and European regulations for the care and use of laboratory animals (Décrets 87-848, 2001-464, 2001-486 and 2001-131 and European Directive 2010/63/UE) and approved by the local ethical committee Comité d'Ethique en matière d'Expérimentation Animale, Universite Paris Descartes, Paris, France. No: CEEA34.GD.002.11. All surgery was performed under anesthesia, and all efforts were made to minimize suffering. For human skin, written informed consent was obtained and all procedures were performed according to French national guidelines and approved by the local ethical committee, Comité d'Evaluation Ethique de l'INSERM IRB 00003888 FWA 00005881, Paris, France Opinion: 11-048.

Animals grafting and infection. Humanized SCID/Beige (SOPF/CB17 SCID BEIGE. CB17.Cg-Prkdc-Lyst/Crl) mice (Charles River, France) 5-8 weeks of age were used as described in earlier studies (Melican et al., 2013). Briefly, anesthetized mice were grafted with healthy human skin, including epidermis, dermis and dermal microvasculature. Furthermore, 3-4 weeks post graft, the tissue retained human morphology without inflammation, $10^{7} \mathrm{CFU}$ of GFP-expressing bacteria were injected IV and the animals were sacrificed $2 \mathrm{~h}$ post infection.

Preparation of tissue samples for immunohistochemistry. Tissues were fixed with $4 \%$ paraformaldehyde (PFA), frozen in OCT (Tissuetek) and sliced at $10 \mu \mathrm{m}$. Human vessels were stained for plasma membrane with the human specific Ulex europaeus agglutinin (UEA) lectin coupled to rhodamine (Vector Laboratories) and for F-actin with phalloidin coupled to AlexaFluor-568 (Invitrogen). Type IV pili were detected with an in-house generated anti-PilE nanobody coupled to AlexaFluor-647 (AlexaFluor-647-NHS-ester, Invitrogen).

Preparation of tissue samples for transmission electron microscopy. Tissues were fixed with $4 \%$ PFA and $0.5 \%$ glutaraldehyde in $0.1 \mathrm{M}$ phosphate buffer, for $4 \mathrm{~h}$ at room temperature. They were then transferred to $1 \%$ PFA in $0.1 \mathrm{M}$ phosphate buffer and incubated overnight at $4{ }^{\circ} \mathrm{C}$. Samples were next processed as for conventional TEM.

Cell culture. Human umbilical vein endothelial cells (HUVEC, Promocell) were maintained in Endo-SFM (human endothelial-SFM, Gibco) supplemented with $10 \%(\mathrm{v} / \mathrm{v})$ heat-inactivated fetal bovine serum (FBS, PAA Laboratories) containing $10 \mu \mathrm{g} \cdot \mathrm{mL}^{-1}$ endothelial cells growth supplement (ECGS, Alfa Aesar) and were used between passages 3 and 9 .

Bacterial strains and culture. Neisseria meningitidis $(\mathrm{Nm})$ serogroup C strain 8013 is a capsulated, piliated, Opa ${ }^{-} \mathrm{Opc}^{-}$clinical isolate (Nassif et al., 1993). It was grown on GCB-Agar (Difco) plates containing Kellogg's supplements (Kellogg et al., 1968) and antibiotics when required (kanamycin, $100 \mu \mathrm{g} \cdot \mathrm{mL}^{-1}$; chloramphenicol, $5 \mu \mathrm{g} \cdot \mathrm{mL}^{-1}$; spectinomycin, $50 \mu \mathrm{g} \cdot \mathrm{mL}^{-1}$; erythromycin, $2 \mu \mathrm{g} \cdot \mathrm{mL}^{-1}$ ) at $37^{\circ} \mathrm{C}$ and $5 \% \mathrm{CO}_{2}$ under moist atmosphere. For infection experiments, GCB-Agar grown bacteria were resuspended in Endo-SFM supplemented with $10 \%$ FBS at an 
$\mathrm{OD}_{600}$ of 0.05 and cultured for $2 \mathrm{~h}$ with shaking at $37^{\circ} \mathrm{C}$ and $5 \% \mathrm{CO}_{2}$. The GFP expressing strain was described elsewhere (Melican et al. 2013). A plasmid allowing stable expression of the iRFP near-infrared fluorescent protein (Filonov et al., 2011) in $\mathrm{Nm}$, pMGC13, was constructed as follows: the sequence encoding the iRFP protein was PCR-amplified from the plasmid $\mathrm{pBAD} / \mathrm{His}-\mathrm{B}$-iRFP (a kind gift from Vladislav Verkhusha, Addgene plasmid \#31855) with PacI and SalI restriction sites in 5' and ' respectively and the ribosome binding site from $\mathrm{Nm}$ pilE gene just upstream of the start codon. The sequences for the primers are iRFP_F2: TTAATTAAAGGAGTAATTTTATGGGGGGTTCTCATCATCATCA and iRFP_R3: GTCGACTCACTCTTCCATCACGCCGATCTGC (with restriction sites underlined and RBS from pilE in italic). The PCR fragment was then cloned in a pCRII-TOPO vector (Invitrogen), checked for sequence and subcloned in the pMGC5 plasmid that allows homologous recombination at an intergenic locus of the Nm chromosome and expression under the control of the constitutive pilE promoter (Soyer et al., 2014). The pilT-iRFP mutant strain deficient for type IV pili retraction was generated by homologous recombination of the chromosomal DNA of the pilT insertion mutant strain into the wild-type iRFP strain by natural transformation. The non-capsulated $\mathrm{Opa}^{+}$-siaD-iRFP and its non-piliated derivative $\mathrm{Opa}^{+}$-siaD-pilD-iRFP was generated by homologous recombination of the chromosomal DNA from siaD and/or pilD insertion mutant strains into a naturally occurring variant of $2 \mathrm{C} 43$, which carries a opaB gene in the ON phase, by natural transformation.

Cell transfection and plasmids. In all, $5.10^{5}$ HUVEC were electroporated with $4 \mu \mathrm{g}$ plasmid DNA encoding either the enhanced green fluorescent protein fused to palmitoylation and myristoylation signals from the Lyn protein (PM-EGFP, kindly provided by Barbara Baird and David Holowka, Cornell University, Ithaca), a farnesylated GFP (GFP-F, Clonetech), and/or LifeAct-mCherry (kindly provided by Guillaume Montagnac, Institut Curie, Paris) or the Carcinoembryonic antigenrelated cell adhesion molecule 1 (CEACAM1, kindly provided by Alain Servin) with the Amaxa Nucleofector device (Amaxa Biosystem, Lonza).

Oblique illumination (dark field) live cell imaging and high speed analysis. In all, 2,1.10 transfected cells were seeded in 96-well glass bottom plates (Sensoplate, Greiner BioOne) coated with $3 \mu \mathrm{g} \cdot \mathrm{mL}^{-1}$ rat tail type I collagen and $50 \mu \mathrm{g} \cdot \mathrm{mL}^{-1}$ human fibronectin (Sigma-Aldrich) and cultivated without ECGS. The next day, cells were infected with vortexed Endo-SFM-FBS grown bacteria at a MOI of 200 and gently rinsed after $10 \mathrm{~min}$ with Endo-SFM-FBS and screened for presence of adhering bacteria prior imaging. For inhibition of F-actin polymerization, cells were treated with $100 \mathrm{nM}$ cytochalasin D in Endo-SFM-FBS for 20 min at $37^{\circ} \mathrm{C}$ and $5 \% \mathrm{CO}_{2}$ prior to infection. Live imaging was performed on an Eclipse $\mathrm{T} i$ inverted microscope (Nikon) equipped with a laser-based iLas2 Total Internal Reflection Fluorescence microscopy (TIRF) module (Roper Scentific) with lasers at $491 \mathrm{~nm}, 561 \mathrm{~nm}$ and $647 \mathrm{~nm}$ and an ORCA03 digital CCD camera (Hamamatsu). Dark field images were obtained by using the TIRF module to get the illumination lasers with an incident angle of $31.2^{\circ}$ in the widefield mode through an oil immersion 100x Apo TIRF objective with a 1.49 numerical aperture and alternatively an additional $1.5 x$ lens. A $2 \times 2$ binning was used. To perform spatial highresolution acquisition, a fast Z-piezo stage (Piezo Nano Z100) was adapted onto the stage of the microscope. For imaging in the stream mode, images were recorded at the camera frame rate (5 to 6 images per second) for $10 \mathrm{sec}$. Focus was maintained with the Perfect Focus System (PFS, Nikon). All experiments were performed at $37^{\circ} \mathrm{C}$ in an incubation chamber adapted for the microscope (Microscope Temperature Control System, LIS). The set-up was controlled by the MetaMorph software (Molecular Devices). Times of appearance and disappearance of the plasma membrane protrusions around each bacterium were analyzed manually. $k_{\text {on }}$ and $k_{\text {off }}$ were calculated by dividing the total number of appearing or disappearing protrusions in all the bacteria of a given experiment by the total number of protrusions observed and by $10 \mathrm{sec}$, and were consequently expressed in $\mathrm{s}^{-1}$. The mean lifetime for plasma membrane protrusions was calculated as the inverse of $k_{\text {off }}$ and was therefore expressed in seconds.

Oblique illumination live cell imaging of HUVEC on micropatterns. Micropatterning on glass coverslips was performed as previously described ${ }^{62}$. Briefly, after plasma activation (Plasma Cleaner, Harrick), we passivated the glass with PLL-g-PEG (Surface Solutions GmbH, 0,1 mg.mL ${ }^{-1}$ in $10 \mathrm{mM}$ HEPES pH 7.4) for $30 \mathrm{~min}$. After washing with water, we illuminated the surface with deep UV light (UVO Cleaner) through a chromium synthetic quartz photomask (Toppan). For imaging, we used channel-shaped sticky slides (Ibidi) that we bound on top of micropatterned coverslips. The home built chambers were sterilized by $10 \mathrm{~min}$ UV exposure under the hood. Finally, we incubated $1 \mathrm{~h}$ with human fibronectin $(50 \mu \mathrm{g}$. $\mathrm{mL}^{-1}$ in water) and seeded PM-EGFP expressing HUVEC cells at a density of 50,000 cells per channel. $1 \mathrm{~h}$ post seeding, cells were rinsed three times with fresh Endo-SFM to get rid of non-adhering cells, and cultured overnight. For infection, iRFP and mCherry wild-type bacteria from liquid cultures were vortexed and loaded into the channels in a 1:10 ratio to a final MOI of 400. Samples were incubated for $10 \mathrm{~min}$ at $37^{\circ} \mathrm{C}$ and $5 \% \mathrm{CO}_{2}$ and then rinsed thoroughly three times with fresh Endo-SFM to remove non-adhering bacteria. To follow membrane remodeling during bacterial proliferation, oblique imaging with 491, 561 and 642 $\mathrm{nm}$ lasers was performed with a 100X ApoTIRF objective (Z-stacks: $1 \mu \mathrm{m}$ steps over $10 \mu \mathrm{m}$; time-lapse: $10 \mathrm{~min}$ time frame over $10 \mathrm{~h}$ ).

Scanning electron microscopy (SEM). A total of $1,5.10^{5}$ HUVEC infected with $\mathrm{Nm}$ for $10 \mathrm{~min}$ at a MOI of 400 or bacteria alone cultured on $12 \mathrm{~mm}$ glass slides were chemically pre-fixed by addition of one volume of pre-warmed $8 \%$ PFA in 0.1 $\mathrm{M}$ HEPES $\mathrm{pH} 7.4$ and incubated at room temperature for $45 \mathrm{~min}$. After three washing steps in HEPES, type IV pili were immunostained or not after 20 min blacking in HEPES-0.2\% gelatin (HEPES-G) with $1.2 \mu \mathrm{g} \cdot \mathrm{mL}^{-1} 20 \mathrm{D} 9$ antibody and a fluorescent secondary antibody or $2.4 \mu \mathrm{g} \cdot \mathrm{mL}^{-1} 20 \mathrm{D} 9$ antibody and a secondary antibody coupled to $15 \mathrm{~nm}$ colloidal gold particles (EM.GMHL15, BBI Solutions) diluted 1:30. HEPES was used instead of PBS in all steps. Infected cells were then post-fixed with $2.5 \%$ glutaraldehyde in HEPES, overnight at $4{ }^{\circ} \mathrm{C}$, washed in HEPES, post-fixed with $1 \% \mathrm{OsO}_{4}$ in HEPES for $1 \mathrm{~h}$, washed in distilled water, dehydrated in graded series of ethanol $(25,50,75,90$ and $100 \%)$, critical point dried with Leica EM CPD300, mounted on aluminum stubs and sputter-coated with $20 \mathrm{~nm}$ gold/palladium with a Gatan PEC 682 gun ionic evaporator. SEM was performed in an Auriga scanning microscope (Carl Zeiss) operated at $7 \mathrm{kV}$ with an in-lens secondary electrons detector. For immunogold labeled samples, images were simultaneously acquired through the secondary electrons detector and the backscattered electrons detector at $20 \mathrm{kV}$. Colorized images were generated with Adobe Photoshop.

HPF-FS and TEM. $3 \times 0.05 \mathrm{~mm}$ sapphire disks $(\mathrm{M}$. Wohlwend $\mathrm{GmbH})$ were cleaned in 100\% ethanol, carbon coated in a Baltec MED 010 evaporator, baked at $130{ }^{\circ} \mathrm{C}$ for $8 \mathrm{~h}$, glow discharged for $5 \mathrm{sec}$ in a Harrick PDC-32G-2 air plasma cleaner at low radiofrequency and coated immediately with $2.5 \mu \mathrm{g} \cdot \mathrm{mL}^{-1}$ rat tail type I collagen and $50 \mu \mathrm{g} . \mathrm{mL}^{-1}$ human fibronectin. Disks were seeded with $1.5 .10^{5}$ cells in 24-well plates. The next day, cells were infected with $\mathrm{Nm}$ for $2 \mathrm{~h}$ at a MOI of 400 and chemically pre-fixed by addition of one volume of a mix of pre-warmed 8\% PFA and 1\% EM-grade glutaraldehyde in 0.1 M HEPES pH 7.4 and incubated at room temperature for $45 \mathrm{~min}$. Cells were rinsed three times in 0.1 M HEPES. Disks were then spaced by a golden O-ring, sandwiched between a $0.4 \mathrm{~mm}$ and a $0.5 \mathrm{~mm}$ copper spacer, high pressure frozen in a Baltec HPM 010 and stored in liquid nitrogen. Samples were freeze substituted in a RMC Boeckler FS-8500 with a mix of $1 \% \mathrm{OsO} 4,0.1 \%$ uranyl acetate and $5 \%$ water in glass distilled acetone. Freeze substitution cycle was as follows: $-90^{\circ} \mathrm{C}$ for $1 \mathrm{~h}, 2.5^{\circ} \mathrm{C}$ increase per hour for $16 \mathrm{~h}$ $-50{ }^{\circ} \mathrm{C}$ for $30 \mathrm{~min}, 15^{\circ} \mathrm{C}$ increase per $\mathrm{h}$ for $2 \mathrm{~h},-20^{\circ} \mathrm{C}$ for $30 \mathrm{~min}, 10^{\circ} \mathrm{C}$ increase per hour for $2 \mathrm{~h}$ and $0{ }^{\circ} \mathrm{C}$ for $1 \mathrm{~h}$. After four washes in acetone, sample were embedded in Epon (Epon 812, AGAR1031 kit, hard formula, Agar 100). Alternatively, type IV pili were immunogold labelled before embedding. In this case, cells were chemically pre-fixed with $4 \%$ PFA and $0.1 \%$ glutaraldehyde (final concentrations), rinsed three times in HEPES, quenched with $50 \mathrm{mM} \mathrm{NH}_{4} \mathrm{Cl}$ in HEPES for $5 \mathrm{~min}$, blocked with HEPES-G for $20 \mathrm{~min}$, incubated with $12 \mu \mathrm{g} \cdot \mathrm{mL}^{-1}$ 20D9 antibody in HEPES-G for $1 \mathrm{~h}$, washed twice in HEPES-G, incubated with goat anti-mouse antibody coupled to $6 \mathrm{~nm}$ colloidal gold particles (EM.GMHL6, BBI Solutions) diluted 1:30 in HEPES-G for 30 min, washed five times in HEPES-G and post-fixed with $1 \%$ glutaraldehyde in HEPES for $15 \mathrm{~min}$. Samples were then high pressure frozen and freeze substituted as described above. Ultrathin sections of $60 \mathrm{~nm}$ nominal thickness were cut with a Leica EM UC7 ultramicrotome and collected on carbon coated, formvar-supported hexagonal copper grids, contrasted with $4 \%$ uranyl acetate and 3\% Reynold's lead citrate and imaged in a FEI Tecnai T12 transmission electron microscope operated at an accelerating voltage of $120 \mathrm{kV}$.

Immunofluorescence on $\mathbf{2} \mathbf{~ h}$ infected cells. 1.5.10 $0^{5}$ HUVEC were cultured in 96 well glass bottom plates coated with $2.5 \mu \mathrm{g} \cdot \mathrm{mL}^{-1}$ rat tail type I collagen and $50 \mu \mathrm{g}$. $\mathrm{mL}^{-1}$ human fibronectin and infected the next day with $\mathrm{Nm}$ at an MOI of 100 . After 30 min adhesion in the presence of 0,13 or $26 \mu \mathrm{g} \cdot \mathrm{mL}^{-1} 20 \mathrm{D} 9$ antibody, unbound bacteria were washed three times with fresh cell culture medium and allowed to proliferate for $2 \mathrm{~h}$ in the presence or not of the same concentrations of 20D9. Infected cells were then fixed with $4 \% \mathrm{PFA}$ and processed for immunofluorescence as for 3D SIM except that the 20D9 staining was omitted in wells were the antibody was already present. Ezrin was detected with a rabbit polyclonal antiezrin antibody (kind gift of Paul Mangeat). Images were taken with the Nikon T $i$ Eclipse spinning disk through a 100X oil immersion objective and ezrin recruitment was detected manually.

3D structured illumination microscopy (3D-SIM). 1.5.10 $0^{5}$ PM-EGFP expressing cells were seeded onto $12 \mathrm{~mm}$ glass coverslips coated with $3 \mu \mathrm{g} \cdot \mathrm{mL}^{-1}$ rat tail type I collagen and $50 \mu \mathrm{g} \cdot \mathrm{mL}^{-1}$ human fibronectin and cultivated without ECGS. The next day, cells were infected for $30 \mathrm{~min}$ at a MOI of 200 with Endo-SFM-FBS grown bacteria and fixed by addition of one volume of pre-warmed 8\% PFA in PBS and incubation at room temperature for $30 \mathrm{~min}$. Cells were rinsed three times in PBS, permeabilized for 10 min with PBS-0.1\% Triton-X100 and blocked for $30 \mathrm{~min}$ in PBS-0.2\% gelatin (PBSG). Cells were then incubated for $1 \mathrm{~h}$ with $2 \mu \mathrm{g} \cdot \mathrm{mL}^{-1}$ rabbit polyclonal anti-GFP antibody to amplify the membrane GFP signal (A11122, Invitrogen) and $1.2 \mu \mathrm{g} \cdot \mathrm{mL}^{-1}$ mouse monoclonal anti-PilE antibody 20D9 (Pujol et al., 1997) to detect type IV pili. Cells were incubated with $10 \mu \mathrm{g} \cdot \mathrm{mL}^{-1}$ secondary 
goat anti-rabbit and goat anti-mouse polyclonal antibodies coupled to AlexaFluor488 and -568 (Invitrogen) for $1 \mathrm{~h}$. DNA was detected with $0,5 \mu \mathrm{g} . \mathrm{mL}^{-1}$ DAPI. All antibodies and DAPI were diluted in PBSG. Coverslips were mounted in Vectashield mounting medium (Vector Laboratories) and sealed with clear nail polish. SIM was performed on the ELYRA system controlled by the Zen software (Carl Zeiss) equipped with diode lasers at $405 \mathrm{~nm}, 488 \mathrm{~nm}$ and $561 \mathrm{~nm}$. Images were acquired with an oil immersion 100x Plan-Apochromat M27 objective with a 1.46 numerical aperture and an additional 1.6x lens. Nominal pixel size was 0.049 and $0.084 \mu \mathrm{m}$ in the $\mathrm{XY}$ and $\mathrm{XZ}$ planes, respectively, using a $1 \times 1$ binning. Structured illumination grid pattern was shifted by five different phases and three different angles along the whole Z-stack. Super resolved images were computationally reconstructed and channels were aligned with $100 \mathrm{~nm}$ TetraSpeck beads (Invitrogen) mounted in Vectashield. To further avoid reconstruction artefacts, raw images were checked for bleaching using the SIMcheck plugin ${ }^{63}$ in Fiji ${ }^{64}$. Only data sets with total intensity variations below $20 \%$ over the whole Z-stack were used. We could detect however the presence of putative "hammerstroke" artifacts difficult to avoid in SIM ${ }^{65}$. Contrast was linearly enhanced in Fiji for clarity.

Mathematical description of membrane wetting along adhesive fibers. The free energy of the membrane spreading on a cylindrical fiber is the sum of three contributions: curvature energy, surface energy and gain of the binding energy between the adhesion molecules on the membrane and binders on the fiber,

$$
F_{2 \mathrm{D}}=\frac{\pi \kappa_{\mathrm{b}} L}{r}+2 \pi r \sigma L+2 \pi r(-W) L
$$

where $r$ is the radius of the actin fiber, $\kappa_{\mathrm{b}}$ is the bending modulus, $\sigma$ is the membrane tension of the GUV, $L$ is the spreading length of the membrane along the fiber, and $W$ is the adhesion energy per unit area described as

$$
W=\Gamma \cdot U
$$

In this equation, $\Gamma$ is the number of binders per unit area on the fiber and $U$ is the adhesion energy per pair of adhesion molecule-binder.

From the free energy, we derived the driving force of membrane spreading, $f_{\mathrm{d}}=-\frac{\mathrm{d} F_{2 \mathrm{D}}}{\mathrm{d} L}$

$$
f_{\mathrm{d}}=2 \pi r\left(W-\frac{\kappa_{\mathrm{b}}}{2 r^{2}}-\sigma\right)
$$

For membrane spreading to occur, $f_{\mathrm{d}}$ has to be positive, leading to a minimum radius for an adhesive fiber

$$
r_{\min }=\sqrt{\frac{\kappa_{\mathrm{b}}}{2(W-\sigma)}}
$$

On a fiber of radius $r>r_{\min }$, the floppy vesicle spreads. As $L$ increases, the tension of the vesicle increases because the excess membrane area decreases. Thus, the driving force $f_{\mathrm{d}}$ decreases and the spreading stops when $f_{\mathrm{d}}=0\left(\sigma=\sigma_{\mathrm{M}}=W-\frac{\kappa_{\mathrm{b}}}{2 r^{2}}\right)$.

For an adhesive fiber of radius $r<r_{\text {min }}$, the vesicle cannot wrap around it. Instead, a membrane tube grows along the fiber. The free energy of a tubular membrane having a radius $R_{\mathrm{t}}$ growing along the adhesive fiber with a length $L_{\mathrm{t}}$ is the sum of the tube energy $F_{\mathrm{t}}$ and the gain of the adhesion energy $W_{\mathrm{t}} L_{\mathrm{t}}$

$$
F_{1 \mathrm{D}}=F_{\mathrm{t}}-W_{\mathrm{t}} L_{\mathrm{t}}
$$

The tube energy is the sum of the curvature energy and the surface energy,

$$
F_{\mathrm{t}}=\frac{\pi \kappa_{\mathrm{b}} L_{\mathrm{t}}}{R_{\mathrm{t}}}+2 \pi R_{\mathrm{t}} \sigma L_{\mathrm{t}}
$$

and $W_{\mathrm{t}}$ is the adhesion energy of an adhesion molecule-binder pair per unit length, given by

$$
W_{\mathrm{t}}=\Gamma_{1} \cdot U
$$

where $\Gamma_{1}$ is the number of bonds per unit length. $F_{1 \mathrm{D}}$ depends upon two independent variables, $L_{\mathrm{t}}$ and the volume $\Omega=\pi R_{\mathrm{t}}^{2} L_{\mathrm{t}}$. The force is coupled to $L_{\mathrm{t}}$, and the pressure inside the tube to $\Omega^{66}$. The force is classically derived from the total energy $F_{1 \mathrm{D}}$ with respect to $L_{\mathrm{t}}$ at constant volume, $\Omega=\pi R_{\mathrm{t}}{ }^{2} L_{\mathrm{t}}$, and is given by $\left.f_{L}=-\frac{\mathrm{d} F_{10}}{\mathrm{~d} L}\right]$. It leads to

$$
f_{L}=-\frac{3 \pi}{2} \frac{\kappa_{\mathrm{B}}}{R_{\mathrm{t}}}-\pi \sigma R_{\mathrm{t}}+W_{\mathrm{t}}
$$

and the pressure inside the tube $p_{\mathrm{t}}$ by the derivative of the free energy with respect to $\Omega$ at constant length; moreover, $p_{\mathrm{t}}$ calculated from $F_{\mathrm{t}}$ has to be equal to the pressure inside the vesicle, which is approximated to zero ${ }^{29}$. It leads to

$$
p_{\mathrm{t}}=\frac{\sigma}{R_{\mathrm{t}}}-\frac{1}{2} \frac{\kappa_{\mathrm{b}}}{R_{\mathrm{t}}^{3}} \cong 0
$$

These two equations lead to

$$
f_{L}=-2 \pi \sqrt{2 \kappa_{\mathrm{b}} \sigma}+W_{\mathrm{t}}
$$

The first term is the retraction force acting on the tube $e^{26-29,67}$ and the second term is the driving force pulling the tube.

Value of $\boldsymbol{r}_{\min }$ for a GUV spreading on biotinylated actin fibers. Given that the size of a NeutrAvidin molecule is $5 \mathrm{~nm}^{31}$, which is comparable to the diameter of an actin molecule, $4-7 \mathrm{~nm}^{68}$, and the molar ratio of biotinylated actin:actin is $1: 10, \Gamma \approx \frac{1}{10} \times \frac{1}{5^{2} \times 10^{-18}}=4 \times 10^{15} \mathrm{~m}^{-2}$. Taking $U=25 \mathrm{k}_{\mathrm{B}} \mathrm{T}^{31,69}, W \approx 10^{17} \mathrm{k}_{\mathrm{B}} \mathrm{T} \cdot \mathrm{m}^{-2}$ $=4 \times 10^{-4} \mathrm{~J} \cdot \mathrm{m}^{-2}$ Here, for a floppy vesicle, taking $\sigma \sim 10^{-6} \mathrm{~J} \cdot \mathrm{m}^{-2}, W=4 \times 10^{-4}$ $\mathrm{J} \cdot \mathrm{m}^{-2}$ and $\kappa_{\mathrm{b}}=10 \mathrm{k}_{\mathrm{B}} \mathrm{T}^{70}$, we obtained $r_{\min } \approx 7 \mathrm{~nm}$. Assuming a cylindrical actin bundle composed of cylindrical actin filaments, we estimated that there should be at least $4-10$ actin filaments in the actin bundle for membrane spreading to occur by " $2 \mathrm{D}$ " wetting.

For a biotinylated actin fiber of radius $r<r_{\min }$, given the molar ratio of biotinylated actin:actin to be 1:10 and taking into account the helical structure of actin filaments, we estimate $\Gamma_{1} \approx \frac{1}{10} \times 2 \times \frac{1}{5 \times 10^{-9}}=4 \times 10^{7} \mathrm{~m}^{-1}$. Taking $U=25$ $\mathrm{k}_{\mathrm{B}} \mathrm{T}^{31,69}, W_{\mathrm{t}} \approx 10^{9} \mathrm{k}_{\mathrm{B}} \mathrm{T} \cdot \mathrm{m}^{-1}=4 \mathrm{pN}$. Taking value $W_{\mathrm{t}} \approx 4 p N, \kappa_{\mathrm{b}}=10 \mathrm{k}_{\mathrm{B}} \mathrm{T}^{70}$, we find that $f_{L}>0$ if $\sigma<\sigma_{\mathrm{M}}^{t}=\frac{W_{\mathrm{t}}^{2}}{8 \pi^{2} \kappa_{\mathrm{b}}} \sim 4 \times 10^{-6} \mathrm{~N} \cdot \mathrm{m}^{-1}$.

Dynamics of the two regimes. 2D wetting: The dynamic of membrane spreading on the actin fiber with a radius $r$ is characterized by $\dot{L}=\frac{\mathrm{d} L}{\mathrm{~d} t}$ which can be derived from the balance between the driving force $f_{\mathrm{d}}$ and the friction force $f_{\mathrm{s}}$.

$$
f_{\mathrm{s}}=2 \pi r \dot{L}(\eta+k L)
$$

where $\eta$ is a lipid viscosity and $k=\frac{\eta_{0}}{a}$ is friction coefficient, $\eta_{0}$ is a molecular viscosity and $a$ is a molecular length ${ }^{23}$. The friction force $f_{\mathrm{s}}$ is the sum of friction due to the defect at the contact line between the vesicle and the spreading film, and the friction between the spreading membrane and the surface of the fiber. If the first term is dominant, the balance of the driving and friction forces leads to $\dot{L}=\frac{f_{\mathrm{d}}}{2 \pi r \eta}$. If the second term is dominant, the growth is diffusive at short time and $L^{2}=\frac{f_{\mathrm{d}} \cdot t}{2 \pi r k}$

1D wetting: For membrane nanotube growing along the actin fiber, $\dot{L}_{t}$ is determined by the balance between the driving force $f_{L}$ and the friction force $f_{\mathrm{v}}$ introduced by $\mathrm{E}$. Evans ${ }^{26}$

$$
f_{\mathrm{v}}=2 \pi \tilde{\eta} \dot{L}_{t} \ln \left(\frac{R_{\mathrm{v}}}{R_{\mathrm{t}}}\right)
$$

where $\widetilde{\eta}$ is a membrane viscosity, $R_{\mathrm{v}}$ is the radius of the vesicle from where the membrane nanotube with a radius of $R_{\mathrm{t}}$ grows. The origin of this force is due to the slip between the two monolayers at the entry of the tube: due to the cylindrical geometry, the flux of the lipids in the outer leaflet of the tube is larger than that in the inner on ${ }^{26}$. For short tubes, assuming $\sigma$ is small, the balance of the driving force and the friction force leads to

$$
\dot{L}_{t}=\frac{W_{\mathrm{t}}}{2 \pi \widetilde{\eta} \ln \left(\frac{R_{\mathrm{v}}}{R_{\mathrm{t}}}\right)}
$$

From this equation, taking from Fig. 1f, $\dot{L}_{t} \approx 1-0.25 \mu \mathrm{m} / \mathrm{sec}, W_{\mathrm{t}}=10 \mathrm{pN}$ and $\ln \left(\frac{R_{\mathrm{v}}}{R_{\mathrm{t}}}\right) \approx 7$, we obtained for the membrane surface viscosity $\tilde{\eta}$ ranging between $2 \times$ $10^{-7}$ and $10^{-6} \mathrm{~N} \cdot \mathrm{sec} \cdot \mathrm{m}^{-1}$ (Surface Poiseuille, SP), which are of the same order as the surface viscosities measured by R.E.Waugh for vesicles ${ }^{71}$.

Interaction of giant unilamellar vesicles with adhesive nanofibers. Reagents: 1,2-dioleoyl-sn-glycero-3-phosphocholine (DOPC, 850375) and 1,2-distearoyl-snglycero-3-phosphoethanolamine-N [biotinyl(polyethyleneglycol)-2000] (DSPE-

PEG(2000)-biotin, 880129 P) were purchased from Avanti Polar Lipids. BODIPYTR-C5-ceramide, (BODIPY TR ceramide, D7540) was purchased from Invitrogen. Hellmanex ${ }^{\oplus I}$ was purchased from Hellma (9-307-010-3-507). Culture-Inserts 2 Well for self-insertion were purchased from ibidi (Silicon open chambers, 80209). Biotinylated actin was purchased from Cytoskeleton (biotin-actin, AB07). Phalloidin-FluoProbes ${ }^{\circledast} 488$ was purchased from Interchim (phalloidin488, FP-JO2830). $\beta$-casein from bovine milk (> 98\% pure, C6905), methylcellulose (M0512 $4000 \mathrm{cPs}$ ) and other reagents were purchased from Sigma-Aldrich.

Protein purification: Muscle actin was purified from rabbit muscle and isolated in monomeric form in G-buffer ( $5 \mathrm{mM}^{2}$ Tris- $\mathrm{Cl}^{-}, \mathrm{pH} 7.8,0.1 \mathrm{mM} \mathrm{CaCl}_{2}, 0.2 \mathrm{mM}$ 
ATP, $1 \mathrm{mM} \mathrm{DTT}, 0.01 \% \mathrm{NaN}_{3}$ ) as previously described ${ }^{72}$. Recombinant mouse IRSp53 I-BAR domain was purified as previously described ${ }^{73}$.

GUV wetting on actin fibers: All the steps were performed at room temperature. GUVs composed of DOPC supplemented with 0.5 mole\% BODIPY TR ceramide and $0.5-1 \mathrm{~mole} \%$ DSPE-PEG(2000)-biotin were prepared by using polyvinyl alcohol (PVA) gel-assisted method in a sucrose buffer $(200 \mathrm{mM})$ as described previously ${ }^{74}$. Muscle filamentous actin (F-actin) was pre-polymerized in an actin polymerization buffer (F-buffer, $5 \mathrm{mM}$ Tris-Cl-, pH 7.8, $100 \mathrm{mM} \mathrm{KCl}, 0.2 \mathrm{mM}$ EGTA, $1 \mathrm{mM}$ $\mathrm{MgCl}_{2}, 0.2 \mathrm{mM}$ ATP, $10 \mathrm{mM}$ DTT, $1 \mathrm{mM}$ DABCO, $0.01 \% \mathrm{NaN}_{3}$ ) in the presence of biotin-actin at a biotin-actin:actin molar ratio of $1: 10$, for at least $1 \mathrm{hr}$. To visualize actin, F-actin was labeled with an equal molar amount of phalloidin 488 Glass coverslips were bath sonicated with $2 \%$ Hellmanex for at least $30 \mathrm{~min}$, rinsed with MilliQ water, sonicated with $1 \mathrm{M} \mathrm{KOH}$, and finally sonicated with MilliQ water for $20 \mathrm{~min}$. Experimental chambers were assembled by placing a silicon open chamber on the cleaned coverslip. The glass substrate of the chamber was incubated with IRSp53 I-BAR domain proteins for at least $5 \mathrm{~min}$, rinsed with Fbuffer, incubated with $\beta$-casein $\left(5 \mathrm{mg} \cdot \mathrm{mL}^{-1}\right)$ for at least $5 \mathrm{~min}$, rinsed with F-buffer, incubated with F-actin at a concentration of $3 \mu \mathrm{M}$ and in the presence of $0.3 \%$ methylcellulose for at least $15 \mathrm{~min}$, rinsed with F-buffer, and finally incubated with NeutrAvidin $(1 \mu \mathrm{M})$ for at least $10 \mathrm{~min}$ followed by rinsing with F-buffer. GUVs suspension in F-buffer were added into the chamber and incubated for at least 30 min before observation. To favor actin bundle formation, F-buffer was supplemented with around $20 \mathrm{mM} \mathrm{MgCl}_{2}$ when adding F-actin into the chamber. Samples were observed with an inverted spinning disk confocal microscope (Nikon eclipse Ti-E equipped with a 100X oil immersion objective and a CMOS camera, Prime 95B, Photometrics)

Cryo-electron microscopy of vesicles with adhesive nanofibers. All steps were performed at room temperature. Vesicles were prepared by drying $0.3 \mathrm{mg}$ of lipids dissolving in chloroform (DOPC/ 0.5 mole\% DSPE-PEG(2000)-biotin) under nitrogen gas and then vacuum for at least $1 \mathrm{hr}$, followed by adding $300 \mu \mathrm{L}$ of vesicle buffer $(60 \mathrm{mM} \mathrm{NaCl}$ and $20 \mathrm{mM}$ Tris $\mathrm{pH}$ 7.5) for resuspension, and finally a vortex mixing for $10 \mathrm{sec}$.

A $1.5 \mu \mathrm{M}$ of F-actin containing 10 mole\% biotin-actin in F-buffer was incubated with $\mathrm{MgCl}_{2}$ at a final concentration of $20 \mathrm{mM}$ for $20 \mathrm{~min}$ to favor bundle formation, followed by incubating with NeutrAvidin at $0.7 \mu \mathrm{M}$ for $5 \mathrm{~min} .20 \mu \mathrm{L}$ of vesicles were incubated with $20 \mu \mathrm{L}$ of the pre-bundled F-actin for at least $1 \mathrm{hr}$ before vitrification. The samples were vitrified on copper holey lacey grids (Ted Pella) using an automated device (EMGP, Leica) by blotting the excess sample on the opposite side from the droplet of sample for $4 \mathrm{sec}$ in a humid environment ( $90 \%$ humidity). Imaging was performed on a LaB6 microscope operated at 200 kV (Technai G2, FEI) and equipped with a 4 KX 4K CMOS camera (F416, TVIPS). Automated data collection for $2 \mathrm{D}$ imaging were carried out with the EMTools software suite

Cell culture on anodized aluminum oxide (AAO) membranes (or Anodiscs) $13 \mathrm{~mm}$ Whatman Anodiscs with $100 \mathrm{~nm}$ pores (GE Healthcare) were coated for 30 min with $0,1 \mu \mathrm{g} \cdot \mathrm{mL}^{-1}$ APP3 in $10 \mathrm{mM}$ HEPES $\mathrm{pH}$ 7.4, rinsed three times in 0,1 $\mathrm{M}$ HEPES and once with distilled water, and then coated for $30 \mathrm{~min}$ with $100 \mu \mathrm{M}$ BCN-RGD in PBS and rinsed three times in PBS ${ }^{33} .1,5.10^{5}$ HUVEC were seeded per disc and cultivated overnight. The next day, cell culture medium was renewed with or without $100 \mathrm{~nm}$ cytochalasin $\mathrm{D}$ for $20 \mathrm{~min}$ at $37^{\circ} \mathrm{C}$ and cells were fixed in $2.5 \%$ glutaraldehyde (final concentration). Cells were then processed as for SEM.

Preparation of native basal membranes from mouse mesentery. All experiments were conducted in accordance with the European Directive 2010/63/EU and national regulation for the protection of vertebrate animals used for experimental purposes (Decree 2013-118). Mice were kept in the Specific Pathogen Free (SPF) animal house of Institut Curie for breeding. Mesentery was isolated from a twomonths old female C57BL/6 mouse according to previously described protocol ${ }^{75,76}$. Prior to mesentery isolation, the polyester membrane of a $6.4 \mathrm{~mm}$ diameter transwell insert (Corning) was removed using a scalpel. While holding the mouse intestine with tweezers, the mesentery was stretched and glued on the insert using surgical glue ( $3 \mathrm{M}$ Vetbond). Mesentery was washed with cold PBS supplemented with $1 \%$ Antibiotic-Antimycotic (Life Technologies), and incubated with $1 \mathrm{M}$ ammonium hydroxide $\left(\mathrm{NH}_{4} \mathrm{OH}\right.$, Sigma-Aldrich) for $40 \mathrm{~min}$ at RT to remove all cells present in the mesentery. Mesentery was washed three times with PBS supplemented with $1 \%$ Antibiotic-Antimycotic and stored at $4{ }^{\circ} \mathrm{C}$ prior to seeding with HUVEC cells. Basal membrane with and without endothelial cells were processed for SEM as described above.

Estimation of the binding energy for Pseudomonas aeruginosa T4P. We found an AFM study in which rupturing of type IV pili from Pseudomonas aeruginosa was performed 77 . In this article, there are two experiments where the rupture force was measured at two given velocities (see Fig. 5 of the paper). Assuming a single barrier in the energy landscape, we extracted the binding energy $U$ by using the following equation ${ }^{78}$

$$
f \cdot a=U+k T \ln \left(\frac{V}{V_{0}}\right)
$$

where $f$ is the rupture force, $a$ is the molecular length, $U$ is the adhesion energy, $V$ is the loading velocity and $V_{0}$ is a typical thermal velocity (in the order of $10 \mathrm{~m} \cdot \mathrm{s}^{-1}$ ). From the experimental data $f=250 \mathrm{pN}$ for $V=1 \mu \mathrm{m} \cdot \mathrm{s}^{-1}$ and $f=500 \mathrm{pN}$ for $V=$ $10 \mu \mathrm{m} . \mathrm{s}^{-1}$, we found $U \sim 18 \mathrm{k}_{\mathrm{B}} \mathrm{T}$, which is consistent with the claiming of P.-G. de Gennes that for most practical separation experiments $U \sim 15 \mathrm{k}_{\mathrm{B}} \mathrm{T}^{78}$.

Statistical analysis. Data were analyzed in the Prism software (GraphPad). For comparison of exact values measured in multiple individual bacteria over several independent experiments, the data sets were first tested for normality with a D'Agostino \& Pearson test and then tested for statistical difference accordingly, either by an unpaired Mann-Whitney test (no normality) or an unpaired $t$ test (normality). For comparison of mean values pooled over several independent experiments, statistical difference was tested with a paired $t$ test. ${ }^{*}, P<0.05$; ${ }^{* *}, P<$ 0.01 .

\section{Data availability}

The data that support the findings of this study are available in this article and its Supplementary Information files, or from the corresponding author upon request.

Received: 26 July 2018 Accepted: 19 September 2018

Published online: 25 October 2018

\section{References}

1. McMahon, H. T. \& Gallop, J. L. Membrane curvature and mechanisms of dynamic cell membrane remodelling. Nature 438, 590-596 (2005).

2. Parton, R. G. \& del Pozo, M. A. Caveolae as plasma membrane sensors, protectors and organizers. Nat. Rev. Mol. Cell Biol. 14, 98-112 (2013).

3. McMahon, H. T. \& Boucrot, E. Molecular mechanism and physiological functions of clathrin-mediated endocytosis. Nat. Rev. Mol. Cell Biol. 12, 517-533 (2011)

4. Levin, R., Grinstein, S. \& Canton, J. The life cycle of phagosomes: formation, maturation, and resolution. Immunol. Rev. 273, 156-179 (2016).

5. Ridley, A. J. Life at the leading edge. Cell 145, 1012-1022 (2011).

6. Ham, H., Sreelatha, A. \& Orth, K. Manipulation of host membranes by bacterial effectors. Nat. Rev. Microbiol 9, 635-646 (2011).

7. Rossman, J. S. \& Lamb, R. A. Viral membrane scission. Annu Rev. Cell Dev. Biol. 29, 551-569 (2013).

8. Sheppard, D. C. \& Filler, S. G. Host cell invasion by medically important fungi Cold Spring Harb. Perspect. Med 5, a019687 (2014).

9. Charles-Orszag, A., Lemichez, E., Tran Van Nhieu, G. \& Dumenil, G. Microbial pathogenesis meets biomechanics. Curr. Opin. Cell Biol. 38, 31-37 (2016).

10. Dumenil, G. Revisiting the extracellular lifestyle. Cell Microbiol 13, 1114-1121 (2011).

11. Mikaty, G. et al. Extracellular bacterial pathogen induces host cell surface reorganization to resist shear stress. PLoS Pathog. 5, el000314 (2009).

12. Melican, K., Michea Veloso, P., Martin, T., Bruneval, P. \& Dumenil, G. Adhesion of Neisseria meningitidis to dermal vessels leads to local vascular damage and purpura in a humanized mouse model. PLoS Pathog. 9, e1003139 (2013).

13. Join-Lambert, $\mathrm{O}$. et al. Meningococcal interaction to microvasculature triggers the tissular lesions of purpura fulminans. J. Infect. Dis. 208, 1590-1597 (2013).

14. Melican, K. \& Dumenil, G. Vascular colonization by Neisseria meningitidis. Curr. Opin. Microbiol 15, 50-56 (2012).

15. Brandtzaeg, P. \& van Deuren, M. Classification and pathogenesis of meningococcal infections. Methods Mol. Biol. 799, 21-35 (2012).

16. Eugene, E. et al. Microvilli-like structures are associated with the internalization of virulent capsulated Neisseria meningitidis into vascular endothelial cells. J. Cell Sci. 115, 1231-1241 (2002).

17. Soyer, M. et al. Early sequence of events triggered by the interaction of Neisseria meningitidis with endothelial cells. Cell Microbiol 16, 878-895 (2014).

18. Nassif, $X$. et al. Antigenic variation of pilin regulates adhesion of Neisseria meningitidis to human epithelial cells. Mol. Microbiol 8, 719-725 (1993).

19. Brissac, T., Mikaty, G., Dumenil, G., Coureuil, M. \& Nassif, X. The meningococcal minor pilin PilX is responsible for type IV pilus conformational changes associated with signaling to endothelial cells. Infect. Immun. 80, 3297-3306 (2012)

20. Imhaus, A. F. \& Dumenil, G. The number of Neisseria meningitidis type IV pili determines host cell interaction. Embo J. 33, 1767-1783 (2014). 
21. Gill, D. B., Spitzer, D., Koomey, M., Heuser, J. E. \& Atkinson, J. P. Release of host-derived membrane vesicles following pilus-mediated adhesion of Neisseria gonorrhoeae. Cell Microbiol 7, 1672-1683 (2005).

22. Maissa, N. et al. Strength of Neisseria meningitidis binding to endothelial cells requires highly-ordered CD147/beta2-adrenoceptor clusters assembled by alpha-actinin-4. Nat. Commun. 8, 15764 (2017).

23. Borghi, N., Alias, K., de Gennes, P. G. \& Brochard-Wyart, F. Wetting fibers with liposomes. J. Colloid Interf. Sci. 285, 61-66 (2005).

24. Roux, A. et al. A minimal system allowing tubulation with molecular motors pulling on giant liposomes. Proc. Natl Acad. Sci. USA 99, 5394-5399 (2002).

25. Leduc, C. et al. Cooperative extraction of membrane nanotubes by molecular motors. Proc. Natl Acad. Sci. USA 101, 17096-17101 (2004).

26. Evans, E., \& Yeung, A. Hidden dymanics in rapid changes of bilayer shape. Chem. Phys. Lipids 73, 39-56 (1994)

27. Bozic, B., \& Svetina, S. \& Zeks, B. Theoretical analysis of the formation of membrane microtubes on axially strained vesicles. Phys. Rev. E, 55, 5834-5842 (1997).

28. Derényi, I., . \& Jülicher, F. \& Prost, J. Formation and interaction of membrane tubes. Phys. Rev. Lett., 88, 238101 (2002).

29. Rossier, O. et al. Giant vesicles under flows: extrusion and retraction of tubes. Langmuir 19, 575-584 (2003).

30. Mohandas, N. \& Evans, E. Mechanical-properties of the red-cell membrane in relation to molecular-structure and genetic-defects. Annu Rev. Bioph Biom. 23, 787-818 (1994)

31. Noppl-Simson, D. A. \& Needham, D. Avidin-biotin interactions at vesicle surfaces: adsorption and binding, cross-bridge formation, and lateral interactions. Biophys. J. 70, 1391-1401 (1996).

32. Merkel, R., Nassoy, P., Leung, A., Ritchie, K. \& Evans, E. Energy landscapes of receptor-ligand bonds explored with dynamic force spectroscopy. Nature 397, 50-53 (1999).

33. van Dongen, S. F. M., Maiuri, P., Marie, E., Tribet, C. \& Piel, M. Triggering cell adhesion, migration or shape change with a dynamic surface coating. Adv. Mater. 25, 1687-1691 (2013).

34. Datar, A., Bornschlogl, T., Bassereau, P., Prost, J. \& Pullarkat, P. A. Dynamics of membrane tethers reveal novel aspects of cytoskeleton-membrane interactions in axons. Biophys. J. 108, 489-497 (2015).

35. Pontes, B. et al. Cell cytoskeleton and tether extraction. Biophys. J. 101, 43-52 (2011).

36. Kolappan, S. et al. Structure of the Neisseria meningitidis Type IV pilus. Nat. Commun. 7, 13015 (2016).

37. Dai, J. \& Sheetz, M. P. Membrane tether formation from blebbing cells. Biophys. J. 77, 3363-3370 (1999)

38. Sens, P. \& Plastino, J. Membrane tension and cytoskeleton organization in cell motility. J. Phys. Condens Matter 27, 273103 (2015).

39. Brochard-Wyart, F., Borghi, N., Cuvelier, D. \& Nassoy, P. Hydrodynamic narrowing of tubes extruded from cells. Proc. Natl Acad. Sci. USA 103, 7660-7663 (2006).

40. Parida, S. K. et al. Internalin B is essential for adhesion and mediates the invasion of Listeria monocytogenes into human endothelial cells. Mol. Microbiol 28, 81-93 (1998).

41. Isberg, R. R., Voorhis, D. L. \& Falkow, S. Identification of invasin: a protein that allows enteric bacteria to penetrate cultured mammalian cells. Cell $\mathbf{5 0}$ 769-778 (1987).

42. Todd, W. J., Wray, G. P. \& Hitchcock, P. J. Arrangement of pili in colonies of Neisseria gonorrhoeae. J. Bacteriol. 159, 312-320 (1984).

43. Jude, B. A. \& Taylor, R. K. The physical basis of type 4 pilus-mediated microcolony formation by Vibrio cholerae O1. J. Struct. Biol. 175, 1-9 (2011).

44. Krebs, S. J. \& Taylor, R. K. Protection and attachment of Vibrio cholerae mediated by the toxin-coregulated pilus in the infant mouse model. $J$. Bacteriol. 193, 5260-5270 (2011).

45. Gil, H., Benach, J. L. \& Thanassi, D. G. Presence of pili on the surface of Francisella tularensis. Infect. Immun. 72, 3042-3047 (2004).

46. Sajjan, U. S., Sun, L., Goldstein, R. \& Forstner, J. F. Cable (cbl) type II pili of cystic fibrosis-associated Burkholderia (Pseudomonas) cepacia: nucleotide sequence of the cblA major subunit pilin gene and novel morphology of the assembled appendage fibers. J. Bacteriol. 177, 1030-1038 (1995).

47. Alteri, C. J. et al. Mycobacterium tuberculosis produces pili during human infection. Proc. Natl Acad. Sci. USA 104, 5145-5150 (2007).

48. Olsen, A., Jonsson, A. \& Normark, S. Fibronectin binding mediated by a novel class of surface organelles on Escherichia coli. Nature 338, 652-655 (1989).

49. Collinson, S. K., Emody, L., Muller, K. H., Trust, T. J. \& Kay, W. W. Purification and characterization of thin, aggregative fimbriae from Salmonella enteritidis. J. Bacteriol. 173, 4773-4781 (1991).

50. Alcantar-Curiel, M. D. et al. Multi-functional analysis of Klebsiella pneumoniae fimbrial types in adherence and biofilm formation. Virulence 4, 129-138 (2013).

51. Kristich, C. J. et al. Development and use of an efficient system for random mariner transposon mutagenesis to identify novel genetic determinants of biofilm formation in the core Enterococcus faecalis genome. Appl. Environ. Microbiol 74, 3377-3386 (2008).

52. Jarrell, K. F., Ding, Y., Nair, D. B. \& Siu, S. Surface appendages of archaea: structure, function, genetics and assembly. Life (Basel) 3, 86-117 (2013).

53. Perras, A. K. et al. Grappling archaea: ultrastructural analyses of an uncultivated, cold-loving archaeon, and its biofilm. Front Microbiol 5, 397 (2014).

54. Abounit, S. et al. Tunneling nanotubes spread fibrillar alpha-synuclein by intercellular trafficking of lysosomes. EMBO J. 35, 2120-2138 (2016).

55. Costanzo, M. et al. Transfer of polyglutamine aggregates in neuronal cells occurs in tunneling nanotubes. J. Cell Sci. 126, 3678-3685 (2013).

56. Marzo, L., Gousset, K. \& Zurzolo, C. Multifaceted roles of tunneling nanotubes in intercellular communication. Front Physiol. 3, 72 (2012).

57. Sowinski, S. et al. Membrane nanotubes physically connect $\mathrm{T}$ cells over long distances presenting a novel route for HIV-1 transmission. Nat. Cell Biol. 10, 211-219 (2008)

58. Zhu, S., Victoria, G. S., Marzo, L., Ghosh, R. \& Zurzolo, C. Prion aggregates transfer through tunneling nanotubes in endocytic vesicles. Prion 9, 125-135 (2015).

59. Vakonakis, I. \& Campbell, I. D. Extracellular matrix: from atomic resolution to ultrastructure. Curr. Opin. Cell Biol. 19, 578-583 (2007).

60. Ruprecht, V. et al. How cells respond to environmental cues - insights from bio-functionalized substrates. J. Cell Sci. 130, 51-61 (2017).

61. Elkhatib, N. et al. Tubular clathrin/AP-2 lattices pinch collagen fibers to support 3D cell migration. Science 356, eaal4713 (2017).

62. Azioune, A., Storch, M., Bornens, M., Thery, M. \& Piel, M. Simple and rapid process for single cell micro-patterning. Lab Chip 9, 1640-1642 (2009).

63. Ball, G. et al. SIMcheck: a toolbox for successful super-resolution structured illumination microscopy. Sci. Rep. 5, 15915 (2015).

64. Schindelin, J. et al. Fiji: an open-source platform for biological-image analysis. Nat. Methods 9, 676-682 (2012).

65. Demmerle, J. et al. Strategic and practical guidelines for successful structured illumination microscopy. Nat. Protoc. 12, 988-1010 (2017).

66. Helfrich, W. Elastic properties of lipid bilayers: theory and possible experiments. Z. Naturforsch. C. 28, 693-703 (1973)

67. Powers, T. R., Huber, G. \& Goldstein, R. E. Fluid-membrane tethers: minimal surfaces and elastic boundary layers. Phys. Rev. E 65, 041901 (2002).

68. von der Ecken, J. et al. Structure of the F-actin-tropomyosin complex. Nature 519, 114 (2015).

69. Merkel, R., Nassoy, P., Leung, A., Ritchie, K. \& Evans, E. Energy landscapes of receptor-ligand bonds explored with dynamic force spectroscopy. Nature 397 50 (1999).

70. Rawicz, W., Olbrich, K., McIntosh, T., Needham, D. \& Evans, E. Effect of chain length and unsaturation on elasticity of lipid bilayers. Biophys. J. 79, 328-339 (2000).

71. Waugh, R. E. Surface viscosity measurements from large bilayer vesicle tether formation. II. Exp. Biophys. J. 38, 29-37 (1982).

72. Spudich, J. A. \& Watt, S. The regulation of rabbit skeletal muscle contraction I Biochemical studies of the interaction of the tropomyosin-troponin complex with actin and the proteolytic fragments of myosin. J. Biol. Chem. 246, 4866-4871 (1971)

73. Saarikangas, J. et al. Molecular mechanisms of membrane deformation by IBAR domain proteins. Curr. Biol. 19, 95-107 (2009).

74. Weinberger, A. et al. Gel-assisted formation of giant unilamellar vesicles. Biophys. J. 105, 154-164 (2013).

75. Glentis, A. et al. Cancer-associated fibroblasts induce metalloproteaseindependent cancer cell invasion of the basement membrane. Nat. Commun. 8, 924 (2017)

76. Schoumacher, M., Glentis, A., Gurchenkov, V. V. \& Vignjevic, D. M. Basement membrane invasion assays: native basement membrane and chemoinvasion assay. Methods Mol. Biol. 1046, 133-144 (2013).

77. Beaussart, A. et al. Nanoscale adhesion forces of Pseudomonas aeruginosa type IV pili. Acs Nano 8, 10723-10733 (2014).

78. Brochard-Wyart, F. \& de Gennes, P. G. Adhesion induced by mobile binders: Dynamics. P Natl Acad. Sci. USA 99, 7854-7859 (2002).

\section{Acknowledgements}

This work was supported by the French ministry for research and higher education (ACO); the Integrative Biology of Emerging Infectious Diseases (IBEID) laboratory of excellence (GD) and the VIP European Research Council starting grant (GD). We gratefully acknowledge the Imagopole-Citech of Institut Pasteur (Paris, France) as well as the France-BioImaging infrastructure network supported by the French National Research Agency (ANR-10-INSB-04; Investments for the Future), and the Région Ilede-France (program Domaine d'Intérêt Majeur-Malinf) for the use of the Zeiss LSM 780 Elyra PS1 microscope and the Zeiss Auriga scanning electron microscope. The authors greatly acknowledge the Cell and Tissue Imaging (PICT-IBiSA), Institut Curie, member 
of the French National Research Infrastructure France-BioImaging (ANR10-INBS-04), and the PICT-IBiSA Institut Curie (Paris, France). This work was supported by Institut Curie, Fondation pour la Recherche Médicale (FRM FDT20170437130) and Ecole Doctorale Frontières du Vivant (FdV)-Programme Bettencourt (RS), ERC StG grant STARLIN (DMV), Centre National de la Recherche Scientifique (CNRS), and P. B. and F. B.-W. belong to the CNRS consortium CellTiss, to the Labex CelTisPhyBio (ANR-11LABX0038) and to Paris Sciences et Lettres (ANR-10-IDEX-0001-02). F.C.T. was funded by the EMBO Long-Term fellowship (ALTF 1527-2014) and Marie Curie actions (H2020-MSCA-IF-2014, project membrane-ezrin-actin). We thank J. Pernier for discussions, providing actin and advice on actin reconstitution. We thank Rafaele Attia and Jian (Olivier) Shi for technical help. We would like to thank Sven van Teeffelen for discussions and for help with the quantitative analysis of dark field microscopy. We would like to thank Dorian Obino for critical reading of the manuscript.

\section{Author contributions}

A.C.-O. designed, conducted and analyzed most of the experiments, and prepared all the figures. G.D. designed the experiments, analyzed the data and supervised the project. F.-C.T. designed and performed the GUVs on adhesive actin fibers experiments. F.-C.T., F.B.-W. and P.B. performed the mathematical analysis of membrane wetting. D.B. performed and analyzed the micropatterns experiments. V.M. and A.C.-O. performed and analyzed the fluorescence experiments in mice. M.S. and A.C.-O. performed and analyzed the HPF-FS TEM experiments. A.M. and A.C.-O. conducted and analyzed the SEM experiments. A.S. and A.C.-O. performed and analyzed the 3D-SIM experiments. K.M. performed the TEM experiment in the mouse. R.S. prepared the mouse basal membranes. F.-C.T. and A.B. performed the cryo-EM experiments. C.M. constructed the pMGC13 plasmid. S.G. and P.L. produced the nanobody against T4P used in the mice experiments. M.P. contributed to the design of the Anodisc experiments and to the interpretation of the data. A.C.-O., F.-C.T., F.B.-W., P.B. and G.D. wrote the manuscript. All the authors edited the manuscript.

\section{Additional information}

Supplementary Information accompanies this paper at https://doi.org/10.1038/s41467018-06948-x.

Competing interests: The authors declare no competing interests.

Reprints and permission information is available online at http://npg.nature.com/ reprintsandpermissions/

Publisher's note: Springer Nature remains neutral with regard to jurisdictional claims in published maps and institutional affiliations.
Open Access This article is licensed under a Creative Commons Attribution 4.0 International License, which permits use, sharing, adaptation, distribution and reproduction in any medium or format, as long as you give appropriate credit to the original author(s) and the source, provide a link to the Creative Commons license, and indicate if changes were made. The images or other third party material in this article are included in the article's Creative Commons license, unless indicated otherwise in a credit line to the material. If material is not included in the article's Creative Commons license and your intended use is not permitted by statutory regulation or exceeds the permitted use, you will need to obtain permission directly from the copyright holder. To view a copy of this license, visit http://creativecommons.org/ licenses/by/4.0/.

(C) The Author(s) 2018 